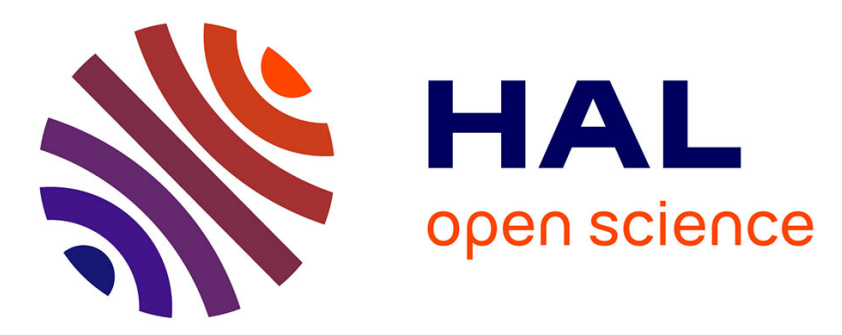

\title{
Innovative blockchain-based farming marketplace and smart contract performance evaluation
}

\author{
Guilain Leduc, Sylvain Kubler, Jean-Philippe Georges
}

\section{To cite this version:}

Guilain Leduc, Sylvain Kubler, Jean-Philippe Georges. Innovative blockchain-based farming marketplace and smart contract performance evaluation. Journal of Cleaner Production, 2021, 306, pp.127055. 10.1016/j.jclepro.2021.127055 . hal-03240813

\section{HAL Id: hal-03240813 \\ https://hal.science/hal-03240813}

Submitted on 28 May 2021

HAL is a multi-disciplinary open access archive for the deposit and dissemination of scientific research documents, whether they are published or not. The documents may come from teaching and research institutions in France or abroad, or from public or private research centers.
L'archive ouverte pluridisciplinaire HAL, est destinée au dépôt et à la diffusion de documents scientifiques de niveau recherche, publiés ou non, émanant des établissements d'enseignement et de recherche français ou étrangers, des laboratoires publics ou privés. 


\title{
Innovative Blockchain-based Farming Marketplace and Smart Contract Performance Evaluation
}

\author{
Guilain Leduc $^{\mathrm{a}, *}$, Sylvain Kubler ${ }^{\mathrm{a}}$, Jean-Philippe Georges ${ }^{\mathrm{a}}$ \\ ${ }^{a}$ Université de Lorraine, CNRS, CRAN, F-54000 Nancy, France
}

\begin{abstract}
E-Agriculture, or Smart Farming, refers to the design, development, and application of innovative methods to use modern information and communication technologies (ICTs), such as the Internet of Things (IoT) and machine learning, to move towards more sustainable agricultural and farming practices. The integration of blockchain technology in farming is gaining attention for its potential to migrate from the centralized and monopolistic model that shapes today's food value chain. This paper highlights the fact that most of today's blockchain-based farming frameworks focus on food tracking and traceability. Only rarely does research focus on the design of digital marketplaces to support the trading of agricultural goods between farmers and potentially interested third party stakeholders; equally rarely are performance evaluations performed for the proposed frameworks. The latter is where this paper contributes the most by, not only proposing a novel blockchain-based farming marketplace platform (called "FarMarketplace"), but also a comprehensive methodology to help software solution integrators to better understand and measure how a given configuration setting of such a platform can influence the overall quality of service performance in the long run.
\end{abstract}

Keywords: Blockchain, Trading, Smart Agriculture, Network Performance, Quality of Service

\section{Introduction}

Agriculture is a sector that is in constant demand. Owing to the increased global population and limited (or scarce) resources, this demand is continuously increasing, enlarging the demand-supply gap (Blandford, 2019). This clearly poses new challenges including the lack of traceability and control throughout the food supply chain, lack of quality assurance, and trust challenges resulting from the growth and consolidation of corporate monopoly power in the food industry (Zhao et al., 2019). Consequently, farmers and the food industry in general are increasingly searching for and adopting new strategies based on modern ICT such as the IoT, cloud computing, big data, and blockchain (Rabah, 2018; Lin et al., 2017; Aker et al., 2016). These emerging technologies have led to the phenomenon of e-agriculture, also referred to as Agriculture 4.0 or smart farming/-

\footnotetext{
${ }^{*}$ Corresponding author

Email addresses: guilain.leduc@univ-lorraine.fr (Guilain Leduc), s.kubler@univ-lorraine.fr (Sylvain Kubler), jean-philippe.georges@univ-lorraine.fr (Jean-Philippe Georges)
}

18 precision $^{1}$ (Lezoche et al., 2020; Wolfert et al., 2017; Vermesan and Friess, 2016), which contributes to making farms more connected, intelligent, and thus more sustainable (Krishnan et al., 2020; Kamble et al., 2019; Klerkx et al., 2019; Rose and Chilvers, 2018).

The emergence of blockchain technology, a distributed ledger technology, has raised significant expectations for moving towards more sustainable farming systems and practices at different levels of the triple bottom line: Social, Environmental, and Economic (Pinto et al., 2019; Tripoli and Schmidhuber, 2018). First, it has the power to break the centralized, monopolistic, asymmetric, and opaque model that shapes today's food value chain (Zhao et al., 2019). Secondly, blockchain offers a unique set of capabilities including decentralization, immutability, transparency, and fault-tolerance, which enables trustless architecture models that were impossible to conceive only a small number of years ago (Bermeo-Almeida et al., 2018; Feng et al., 2020; Rabah, 2018). In recent years, an increasing number of scientific and industrial blockchain-based farming initiatives

\footnotetext{
${ }^{1}$ Although one could argue that distinctions exist between these concepts, we interchangeably use these terms in the remainder of this article.
} 
How does one govern a new economic system?

How do we build trustworthy applications on top of existing blockchain technologies?

How do we scale, increase throughput, speed, fault tolerance, while reducing energy?

What should be the network infrastructure for future blockchain-based ecosystems?

Figure 1: Blockchain Architecture Stack and associated research questions

have appeared around the world (Lin et al., 2017; Kami- ${ }_{8}$ laris et al., 2019), wherein different research questions 82 at the hardware, software, network, and governance levels have been addressed.

Whereas the majority of the research has focused 85 on investigating blockchain-based solutions for the enhanced tracking and traceability of agricultural goods, as is further analyzed and discussed in Section 2, limited research has been undertaken on the design of innovative smart farming digital marketplaces to support the trading of agricultural goods between farmers and interested third party stakeholders (e.g., food transformation companies, retailers, and other farmers). To overcome this gap in research, this paper presents a novel digital marketplace called "FarMarketplace". FarMarketplace fully exploits the advantages of blockchain capabilities by proposing generic, yet detailed representations of trading (smart) contract templates between farmers, interested third-party consumers, and deliverers. Compared with the current literature, FarMarketplace is innovative in three respects.

- The evaluation of this blockchain solution facilitates a methodology to benchmark a blockchain system. It focuses most notably on the allowable capacity offered by the blockchain immediately before saturation. Hence, this evaluation is based on the expected contract emission throughput and its latency according to the block size. Consequently, the notion of capacity, the maximum throughout that the chain can support is introduced. Phenomena around the capacity are also presented, and the methodology is applied for a specific contract.

- Only limited blockchain-based farming frameworks/ecosystems focus on trading and propose any kind of digital marketplaces where farmers/industries, deliverers, and retailers can discover each other and trade agricultural goods and delivery services;

- The majority of the studies do not provide sufficient details regarding the performance of their proposed system (more than $50 \%$ do not evaluate any metrics), and to the best of our knowledge, no study has ever defined a comprehensive methodology to assist software solution integrators understand the performance characteristics and long-run capacity limits - from a quality of service $(Q o S)$ standpoint - of FarMarketplace-like platforms.

The FarMarketplace specifications and performance assessment methodology are detailed in Section 3. The performance evaluation of FarMarketplace is presented in Section 4. The conclusion follows.

\section{Blockchain-based Smart Farming}

A brief overview of the blockchain-related background is given in Section 2.1. In Section 2.2, past and ongoing blockchain-based farming/agricultural initiatives are reviewed and analyzed. Based on this literature review, Section 2.3 discusses the extent to which our research advances the current state-of-the-art.

\subsection{Blockchain background and positioning}

Increasing attention has been devoted to blockchain over the past years as it offers powerful tamper-proof logging and auditing capabilities where trust and control are no longer centralized and black-boxed, but rather decentralized and transparent (i.e., no requirement for a central trusted authority) (Zheng et al., 2018; Panarello et al., 2018). The possibility of defining/using "Smart contract" has opened a wide spectrum of applications where blockchain technology can be leveraged, and identified an entire new class of business models for shared data (Nowiński and Kozma, 2017). In this respect, a number of consortia are working on the design of decentralized digital marketplaces in different sectors such as healthcare, logistics, energy, construction, agriculture, and telecommunication (Al-Jaroodi and Mohamed, 2019). Domain-independent initiatives are also being identified, such as Trusted IoT Alliance ${ }^{2}$ and IOTA Foundation ${ }^{3}$, Enterprise Ethereum Alliance (EEA), and Flowchain ${ }^{4}$. All these initiatives promote

\footnotetext{
${ }^{2}$ https://www.trusted-iot.org, last access Apr. 2020

${ }^{3}$ https://www.iota.org, last access Apr. 2020

${ }^{4}$ https://flowchain.co, last access Apr. 2020
} 
and investigate different, yet common architectural de- 162 sign principles and best practices to achieve specific re- ${ }_{163}$ quirements. These challenges occur at multiple layers 164 of the blockchain stack, as emphasized in Figure 1.

"Consensus" and "(Smart) Contract" are the most 166 discussed layers; the former allowing the secure updating of a distributed shared state, the latter allowing the implementation of user-defined operations of arbitrary complexity that are not possible through plain cryptocurrency protocols such as bitcoin. However, the Consensus layer is undoubtedly the one that has the most influence on network performance, which is strongly dependent on the type of consensus supporting the se- 173 lected/implemented blockchain technology. Consensus 174 protocols are typically grouped into one of three cat- 175 egories: (i) Permissionless (Public): anyone can join, 176 transact, and review the chain without a specific iden- 177 tity; there is no censorship method; (ii) Permissioned (Private): a type of permission is required to access all or part of the blockchain; (iii) Federated (Consortium): this is a hybrid between the two previous groups. 180 Whereas permissionless blockchains are highly scal- 181 able, fault-tolerant, and persistent, they suffer from poor 182 performance with high latency, low throughput, and ${ }_{183}$ high-energy consumption. The opposite applies to permissioned blockchains. It is thus important for software solution integrators to be aware of the extent to which a given blockchain technology influences the overall application performance.

\subsection{Current status of affairs of blockchain-based farm- ing solutions}

A number of blockchain-based agricultural solu- ${ }^{192}$ tions and platforms are emerging throughout the world (Juma et al., 2019), from startup developments such as Skuchain $^{5}$, Provenance ${ }^{6}$, AgriDigital ${ }^{7}$, (Xu et al., 2019) and Farm Share ${ }^{8}$ to larger companies such as Cargill ${ }_{196}$ Risk Management (Dujak and Sajter, 2019).

Even though blockchain is used for different purposes such as minimizing unfair pricing, product origins, and ${ }_{199}$ reducing multinational agricultural influence in favor of ${ }_{200}$ more localized economies (Hang et al., 2020; Galvez 201 et al., 2018; Thomason et al., 2018), its primary objective is to improve transparency and traceability through- 202 out the food chain (Feng et al., 2020; Zhao et al., 2019; ${ }^{203}$ Tripoli and Schmidhuber, 2018). Figure 2 provides an ${ }^{204}$

\footnotetext{
${ }^{5}$ http://www.skuchain.com/, last accessed May 2020.

${ }^{6}$ https://www.provenance.org/, last accessed May 2020

${ }^{7}$ https://www.agridigital.io/, last accessed May 2020.

${ }^{8}$ http://farmshare.org, last accessed May 2020.
}

overview of a traditional food chain, including the contracts that are typically established between the involved parties (Feng et al., 2020; Bumblauskas et al., 2020; Kamilaris et al., 2019). These contracts include the following:

- F2D (Farmer-to-Deliver) and I2D (Industry-toDeliver): contract terms regarding, among other things, the farming or processed food environments, origin of drug variety and processed foods, fertilizing, and product distribution requirements (e.g., cold chain);

- D2F (Deliver-to-Farmers), D2I (Deliver-toIndustry) and D2R (Deliver-to-Farmers): contract terms regarding product distribution including distribution warehousing, delivery, expected product recipient (retailer or industry);

- $R 2 D$ (Retailer-to-Customer: contract terms regarding sales time, price, and quality.

In Table 1, we review and classify the state-ofthe-art studies that consider and eventually implement blockchain technology for smart farming purposes. The papers are classified based on five criteria:

1. Objective: we report why blockchain is used in the study (e.g., for traceability, tracking, trading). Even if traceability and tracking are sometimes used interchangeably, a difference can be made. In a tracing system, the information flow moves backwards through the supply chain (from consumers to suppliers), whereas tracking follows the information forward (from the source to end users) (Laux and Hurburgh Jr, 2012);

2. Smart contract support and focus: we report whether the study makes use of smart contract(s), and if so, we indicate (i) if those contracts are formalized in the corresponding paper ("F" and "N/F" in Table 1 being the respective abbreviations for "Formalized" and "Non-Formalized") and (ii) what chain parties are involved based on the previously introduced contract taxonomy: F2D/I2D, D2F/D2I, D2R, or R2C;

3. Platform: we report whether the study has considered/used an "off-the-shelf" blockchain technology/platform such as Ethereum or Hyperledger;

4. Performance: we report whether the study has performed and detailed any performance evaluation regarding the proposed solutions, whether in terms of time execution, network latency, throughput, security, or other factors. 


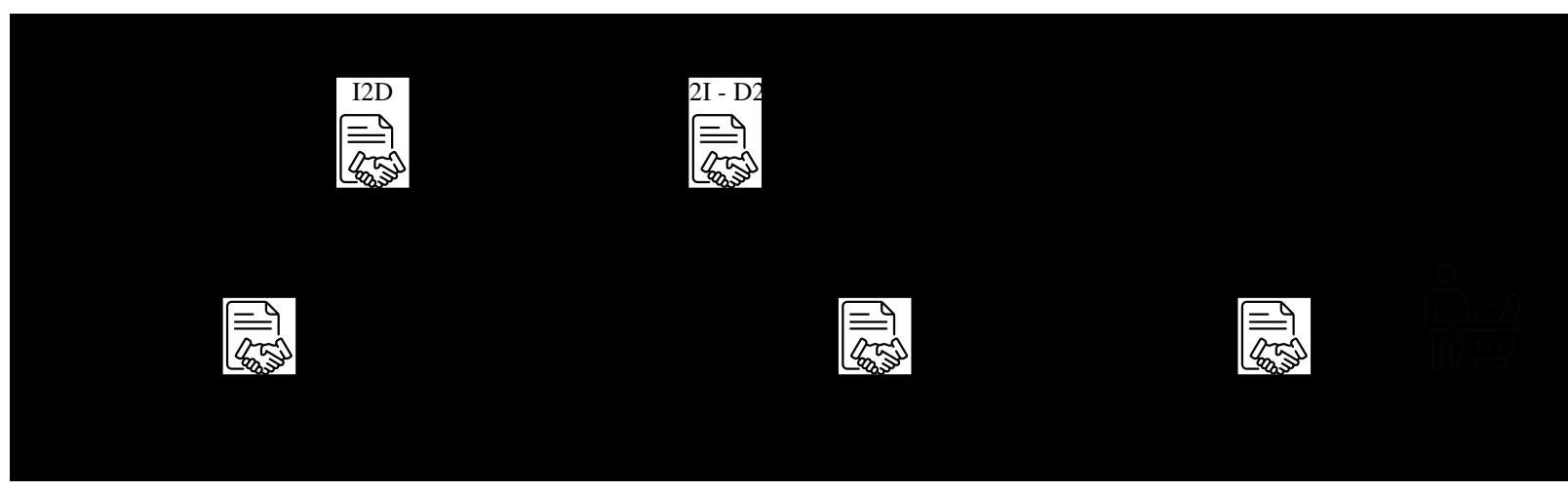

Figure 2: Traditional food chain

First, it can be observed that all the reported stud- 246 ies have been published in the last three years, which ${ }_{247}$ confirms the growing attention paid to blockchain in ${ }_{248}$ the agricultural sector. Moreover, the majority of the 249 studies (23 out of the 27 reported in Table 1) employ 250 blockchain for food traceability and/or tracking pur- 251 poses. The four other studies use blockchain to au- 252 tomate temporary employment contracts between the ${ }_{253}$ farmers and labor contractors (Pinna and Ibba, 2018) 254 and allow for agricultural resource trading between 255 farmers, deliverers, and retailers (Leng et al., 2018; Mao 256 et al., 2019; Bore et al., 2020).

Secondly, virtually all the reported studies exploit ${ }^{258}$ the smart contract capabilities to achieve the above- ${ }^{259}$ mentioned objectives (i.e., to meet traceability, tracking, and trading requirements); 17 of the 27 studies focus on - or fulfill to be more precise - market interactions between farmers/industries, deliverers, and retailers (i.e., F-I2D, D2F-I, R2C). Of these 17 studies, 12 extend the traceability, tracking, or trading facilities to the entire food lifecycle (i.e., covering R2C interactions). It should be noted that the reported studies do not necessarily track/trace the same food system features. Indeed, certain studies such as (Hang et al., 2020; Bumblauskas et al., 2020; Devi et al., 2019; Surasak et al., 2019; Lin et al., 2018) track the environmental background information of a food item using sensor-like devices (e.g., amount of pesticides used, temperature evolution), whereas other studies track other supply chain information such as (i) incident details throughout the crop harvesting process (Iqbal and Butt, 2020), (ii) carbon footprint at food production and transportation levels (Shakhbulatov et al., 2019), and (iii) food quality evolution (Carbone et al., 2018; George et al., 2019).

Thirdly, reviewing what blockchain technologies 280 have been considered in the reported studies (see col- 281 umn "Platform" in Table 1), Ethereum and Hyperledger Fabric are the most widely adopted solutions (the former being used in five studies, the latter in five). This is not surprising as they are both market share leaders $(50 \%$ of the implemented projects being hosted on these platforms) (Udokwu et al., 2018). However, interestingly, one could question why studies aiming to achieve similar goals opt for one or the other? Indeed, whereas Ethereum is more suitable for permissionless distributed ledgers, Hyperledger is more suited to permissioned blockchains (Xie et al., 2019; Sajana et al., 2018). To answer this question, a more in-depth analysis of these studies should be performed to identify the exact system requirements and constraints.

Finally, it can be observed in Table 1 (see column "Performance") that less than half of the reviewed studies performed experimental evaluations of their solutions. In our opinion, this clearly indicates that blockchain-based farming remains in its infancy, where the focus is more on architectural and functional design choices than on performance benchmarking. For studies evaluating the performance of their solution, throughput and latency are the most used metrics, considered in $65 \%$ and $50 \%$, respectively, of the reviewed literature). Throughput corresponds to the number of successful transactions per second (a transaction being successful if it has been validated and committed to a new block); latency corresponds to the delay between the emission of a transaction and its commitment to a new block. It can be observed that only a small number of studies evaluated security aspects. The main reason for this is that the majority of the proposed solutions rely on off-the-shelf blockchain solutions, whose security performance - which is characterized by the number of trusted participants required to secure the blockchain - has been widely studied and described in the literature 
Table 1: Current state of affairs of Smart Farming initiatives

\begin{tabular}{|c|c|c|c|c|c|c|c|c|}
\hline \multirow[t]{2}{*}{ Reference } & \multirow[t]{2}{*}{ Objective } & \multicolumn{5}{|c|}{ Smart Contract (SC) support \& focus } & \multirow[t]{2}{*}{ Platform } & \multirow[t]{2}{*}{ Performance } \\
\hline & & $S C s$ & $(F-I) 2 D$ & $D 2(F-I)$ & $D 2 R$ & $\mathrm{R} 2 \mathrm{C}$ & & \\
\hline (Pinna and Ibba, 2018) & Temp employ. & $\mathrm{F}$ & $\square$ & $\square$ & $\square$ & $\square$ & Ethereum & - \\
\hline (Devi et al., 2019) & Track & $\mathrm{N} / \mathrm{F}$ & $\square$ & $\square$ & $\square$ & $\square$ & Ethereum & 1. Latency \\
\hline (Patil et al., 2017) & Track & - & $\bigotimes$ & $\square$ & $\square$ & $\square$ & $\mathrm{N} / \mathrm{S}$ & - \\
\hline (Tse et al., 2017) & Trace & - & $\bigotimes$ & $\square$ & $\square$ & $\square$ & $\mathrm{N} / \mathrm{S}$ & - \\
\hline (Carbone et al., 2018) & Track & $\mathrm{N} / \mathrm{F}$ & $\bigotimes$ & $\square$ & $\square$ & $\square$ & Hyperledger & - \\
\hline (Hang et al., 2020) & Track & $\mathrm{F}$ & $\bigotimes$ & $\square$ & $\square$ & $\square$ & $\begin{array}{l}\text { Hyperledger } \\
\text { (v1.4.3) }\end{array}$ & $\begin{array}{l}\text { 1. Throughput } \\
\text { 2. Latency }\end{array}$ \\
\hline (Lin et al., 2017) & Trace \& Track & $\mathrm{N} / \mathrm{F}$ & $\bigotimes$ & $\square$ & $\square$ & $\square$ & - & - \\
\hline (Lucena et al., 2018) & Track & $\mathrm{N} / \mathrm{F}$ & 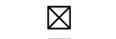 & 凶 & $\square$ & $\square$ & Hyperledger & - \\
\hline (Mao et al., 2019) & Trade & $\mathrm{N} / \mathrm{F}$ & $\bigotimes$ & 凶 & 凶 & $\square$ & Own (FTSCON) & $\begin{array}{l}\text { 1. Exec. time } \\
\text { 2. Merchant profit, } \\
\text { 3. Security }\end{array}$ \\
\hline (Tian, 2017) & Trace & $\mathrm{N} / \mathrm{F}$ & $\bigotimes$ & $\bigotimes$ & $\bigotimes$ & $\square$ & Ethereum & - \\
\hline (Bore et al., 2020) & Trade & $\mathrm{N} / \mathrm{F}$ & $\bigotimes$ & 凶 & 凶 & $\square$ & Hyperledger & $\begin{array}{l}\text { 1. Throughput } \\
\text { 2. Latency }\end{array}$ \\
\hline $\begin{array}{l}\text { (Stefanova and Salampasis, } \\
\text { 2019) }\end{array}$ & Trace & $\mathrm{N} / \mathrm{F}$ & $凶$ & $\bigotimes$ & $\bigotimes$ & $\square$ & Hyperledger & - \\
\hline (Leng et al., 2018) & Trade & $\mathrm{N} / \mathrm{F}$ & 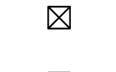 & $\bigotimes$ & $\bigotimes$ & $\square$ & $\mathrm{N} / \mathrm{S}$ & $\begin{array}{l}\text { 1. Throughput } \\
\text { 2. Latency }\end{array}$ \\
\hline (Kumar and Iyengar, 2017) & Trace \& Track & $\mathrm{N} / \mathrm{F}$ & 凶 & $\bigotimes$ & 凶 & $\square$ & - & - \\
\hline (Iqbal and Butt, 2020) & Track & $\mathrm{N} / \mathrm{F}$ & $\bigotimes$ & 凶 & $\bigotimes$ & $\square$ & - & 1. ZigBee-related \\
\hline (George et al., 2019) & Trace \& Track & $\mathrm{N} / \mathrm{F}$ & $\bigotimes$ & 凶 & $\bigotimes$ & $\bigotimes$ & - & - \\
\hline (Caro et al., 2018) & Trace \& Track & $\mathrm{N} / \mathrm{F}$ & $\bigotimes$ & $\bigotimes$ & 凶 & 凶 & $\begin{array}{l}\text { Ethereum \& } \\
\text { Hyperledger }\end{array}$ & $\begin{array}{l}\text { 1. Throughput } \\
\text { 2. Latency } \\
\text { 3. CPU }\end{array}$ \\
\hline (Surasak et al., 2019) & Trace \& Track & $\mathrm{N} / \mathrm{F}$ & $\bigotimes$ & $凶$ & $\bigotimes$ & $\bigotimes$ & SQL-based & - \\
\hline (Bumblauskas et al., 2020) & Trace \& Track & $\mathrm{N} / \mathrm{F}$ & $\bigotimes$ & 凶 & $\bigotimes$ & $\bigotimes$ & Hyperledger & - \\
\hline (Malik et al., 2018) & Trace & $\mathrm{F}$ & $\bigotimes$ & $\bigotimes$ & $\bigotimes$ & $\bigotimes$ & Hyperledger & 1. Time \\
\hline (Hua et al., 2018) & Trace \& Track & $\mathrm{F}$ & $\bigotimes$ & $\bigotimes$ & $\bigotimes$ & $\bigotimes$ & $\mathrm{N} / \mathrm{S}$ & - \\
\hline (Shakhbulatov et al., 2019) & Track & $\mathrm{F}$ & $\bigotimes$ & $\bigotimes$ & 凶 & 凶 & $\begin{array}{l}\text { Raft-like consen- } \\
\text { sus }\end{array}$ & $\begin{array}{l}\text { 1. Throughput } \\
\text { 2. Time }\end{array}$ \\
\hline (Lin et al., 2018) & Trace & $\mathrm{N} / \mathrm{F}$ & $\bigotimes$ & $\bigotimes$ & $\bigotimes$ & $\bigotimes$ & - & - \\
\hline (Reddy and Kumar, 2020) & Trace \& Track & $\mathrm{N} / \mathrm{F}$ & $\bigotimes$ & $\bigotimes$ & $\bigotimes$ & $\bigotimes$ & - & - \\
\hline (Xie et al., 2017) & Track & $\mathrm{F}$ & $\bigotimes$ & $\bar{\nabla}$ & 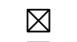 & 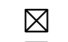 & Ethereum (v1.9) & 1. Throughput \\
\hline (Papa, 2017) & Trace & $\mathrm{N} / \mathrm{F}$ & $\bigotimes$ & $\bigotimes$ & $\bigotimes$ & $\bigotimes$ & - & - \\
\hline (Awan et al., 2019) & Trace \& Track & $\mathrm{N} / \mathrm{F}$ & $\bigotimes$ & $\bigotimes$ & $\bigotimes$ & $\bigotimes$ & $\mathrm{N} / \mathrm{S}$ & 1. Throughput \\
\hline
\end{tabular}

(Ali et al., 2018; Xiao et al., 2020). In fact, the security 299 level of a given blockchain technology is directly de- 300 rived from the consensus protocol supporting the chain. 301 For example, in proof-of-work (PoW) consensus, the ${ }_{302}$ number of honest miners must be greater than $51 \%$; this number must be $\geq 66 \%$ in Byzantine fault toler- 303 ance (BFT) consensus algorithms Vukolić (2015). As 304 a general remark, the current literature does not ad- 305 dress sufficient attention to properly analyzing the extent to which a given architectural design choice can influence the long-run capacity limits (in transactions/second $\mathrm{Tx} / \mathrm{s}$ ) of the proposed system, and hence by definition, on the overall (end-to-end) QoS. As an example, latency is directly dependent on the throughput, and can ${ }_{31}$ be negatively influenced by a high delay of transaction propagation. Furthermore, if the throughput is less than 312 the transaction asking rate, congestion is likely to occur, ${ }^{313}$ which results in an increase in latency. Such interactions between blockchain- and infrastructure-related parameters are rarely analyzed and considered in the literature, thus requiring further research.

\subsection{Positioning and Contribution of this research work}

Based on the literature review presented in the previous section, we stress three important facts.

1. The vast majority of the studies are dedicated to traceability and tracking along supply chains, and conversely, only a limited number focus on trading, i.e., digital marketplaces where farmers/industries, deliverers, and retailers can discover each other and trade agricultural goods and delivery services.

2. The vast majority of the studies use on-the-shelf blockchain technologies; in particular, Ethereum or Hyperledger Fabric. 


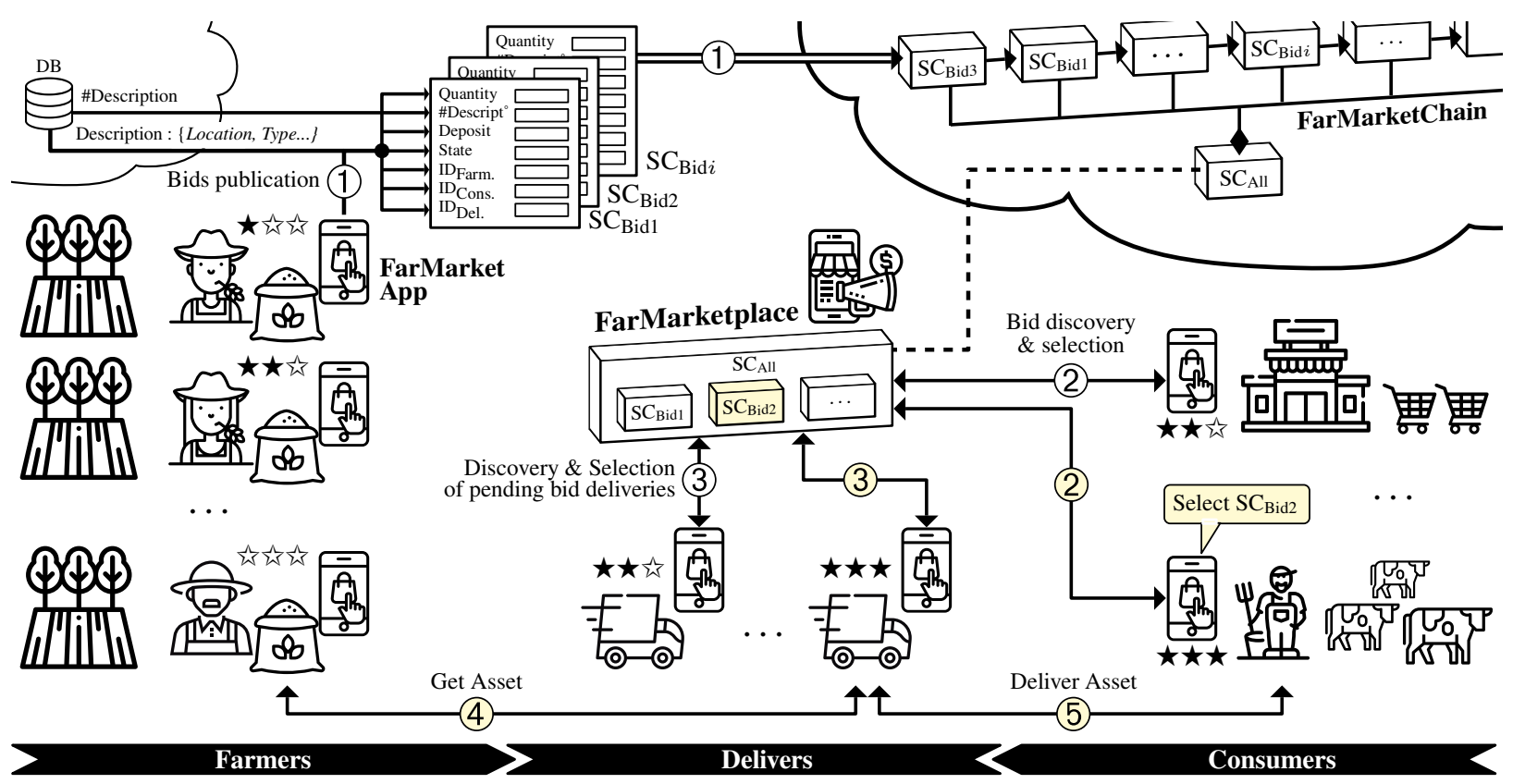

Figure 3: Overview of the FarMarket ecosystem and associated interactions between stakeholders and supporting marketplace

3. The vast majority of the studies do not provide sufficient details regarding the performance of the proposed system (more than 50\% of the studies do 340 not evaluate any metrics). This lack of compre- 341 hensive evaluation in the studies, combined with ${ }_{342}$ the lack of details regarding the implemented smart ${ }_{343}$ contracts (only $20 \%$ of the studies provide rel- ${ }^{344}$ evant details) and lack of details regarding the ${ }_{345}$ blockchain configuration, which has a direct im- 346 pact on the overall system performance, makes ${ }_{347}$ it difficult to compare existing blockchain-based farming frameworks.

Given the above findings, this paper advances the current state-of-the-art in two respects. First, a novel digital marketplace for agricultural product trading purposes is proposed, which is in agreement with research by Mao et al. (2019); Bore et al. (2020); Leng et al. (2018). Secondly, a representation of the interactions that occur between blockchain- and/or infrastructure-related parameters is presented. This representation not only provides software solution integrators with a holistic overview of 359 possible interactions, but also facilitates the analysis of 360 the system (QoS) performance limitations in the long ${ }_{361}$ run.

\section{FarMarket ecosystem}

The digital FarMarletplace proposed in this study is a part of a larger ecosystem referred to as "FarMarket". The building blocks supporting this ecosystem are presented in Section 3.1 and the smart contract templates specified for trading are further detailed in Section 3.2. In Section 3.3, key performance indicators in the performance evaluation process of a FarMarket-like ecosystem are discussed.

\subsection{Ecosystem services and supporting architecture}

An overview of the different stakeholders and software/hardware components supporting the FarMarket ecosystem is depicted in Figure 3. This ecosystem is designed to:

- collect agricultural bids/contracts published by farmers or other like-minded providers (see (1) in Figure 3);

- notify consumers that new bids/contracts are available, allowing them to select/purchase one or more contracts/bids (see (2);

- notify deliverers that new delivery offers are available, allowing them to select one or more offers (see (3), which - if accepted by the farmer and consumer - implies that the deliverer must collect and 
deliver the asset associated to the bid/contract (see (4) and (5)), upon which they will be paid;

- allow all stakeholders to evaluate the service quality, namely (i) the consumer can evaluate the quality of the delivery service (e.g., punctuality, professionalism) and the received agricultural goods, (ii) the deliverer can evaluate the quality of the farmer and consumer (e.g., punctuality, accuracy of the specified location); and (iii) the farmer can evaluate the quality of the delivery service.

To achieve the above functionalities, three main building blocks have been designed and integrated into the FarMarket ecosystem.

1. FarMarketchain: This refers to the blockchain and associated smart contracts. A database, denoted by DB in Figure 3, functions with blockchain to avoid storing long chains of characters in the blockchain itself, which is costly (only the hash of the corresponding chain is added to the blockchain).

2. FarMarketplace: This refers to the digital market- 409 place platform. It hosts the blockchain and DB, 410 and has the role of intermediary between the dif- ${ }_{411}$ ferent ecosystem stakeholders.

3. FarMarketApp: This refers to the App that allows stakeholders to benefit from the set of services offered by the FarMarket ecosystem.

Using an exterior database to store data is a common practice in blockchain development. Indeed, it may become expensive to store raw information in a distributed ledger, as each transaction usually implies a fee, and storing only the hash of information stored in a database (allowing for verifying the data integrity by comparing that hash at any given time) is a widely adopted alternative. This database is, in our case, a server but can be substituted by a private cloud Sumathi et al (2020) or IPFS (InterPlanetary File System) to allow for fully decentralized peer-to-peer framework Singh et al (2019). As the access control of data is not the main focus of this paper, all data is freely accessible, although more advanced access control strategies could be adopted in the future, as defining a XACML politic Ramli et al (2014) or adopting a blockchain-based solution Maesa et al (2017); Esposito et al (2021).

\subsection{Smart farming contracts}

The set of interactions (or communications) occurring between the previously introduced building blocks ${ }_{438}$
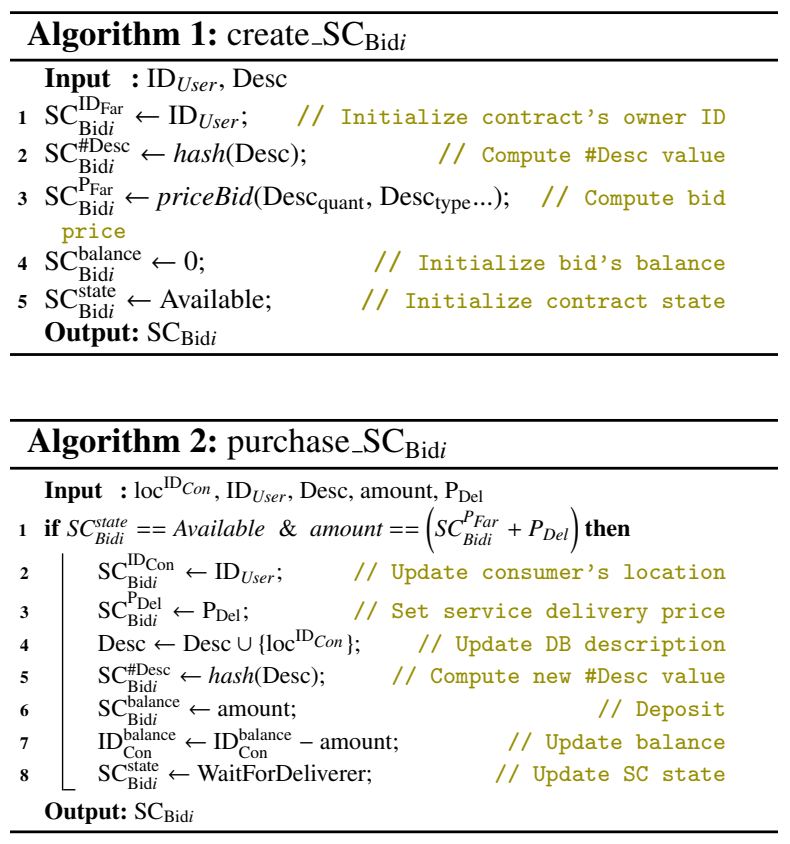

and stakeholders are further detailed in Figure 4 in the form of a sequence diagram.

First, consumers and deliverers can look for bids and pending delivery offers ( $c f ., \mathbf{0}$ in Figure 4), and possibly subscribe to the FarMarketplace platform to be notified whenever a new bid/offer is published (the Message Queuing Telemetry Transport protocol is being used in this respect). Farmers can publish new bids by specifying - via the FarMarketApp - information related to their bid $(c f .$, (2). This action calls the bid creation function detailed in Algorithm 1, where $\mathrm{SC}_{\mathrm{Bid} i}$ refers to a given smart contract ( $i$ referring to the $i^{\text {th }}$ contract). In fact, two input parameters are sent by the FarMarketApp to Algorithm 1, namely, (i) Farmer's ID and (ii) Description (consisting of several information items as detailed in Table 2). Based on these two input parameters, five immutable attributes - also referred to as "state variables" - are extracted/derived to be stored in the FarMarketchain, namely: (i) Farmer's ID, (ii) hash value of the bid description, (iii) bid price, (iv) contract balance, and (v) contract state. The input parameters communicated by FarMarketApp and derived state variables are summarized in Table 2.

At this stage, the contract is available on the marketplace and consumers have been notified of its existence (3). When a consumer selects a bid for purchase ( $c f$., (4), the purchase function of $\mathrm{SC}_{\mathrm{Bid} i}$ is executed, as detailed in Algorithm 2. The purpose of this function/algorithm is to verify that the contract is in the correct state (should be Available for purchase) and that the 


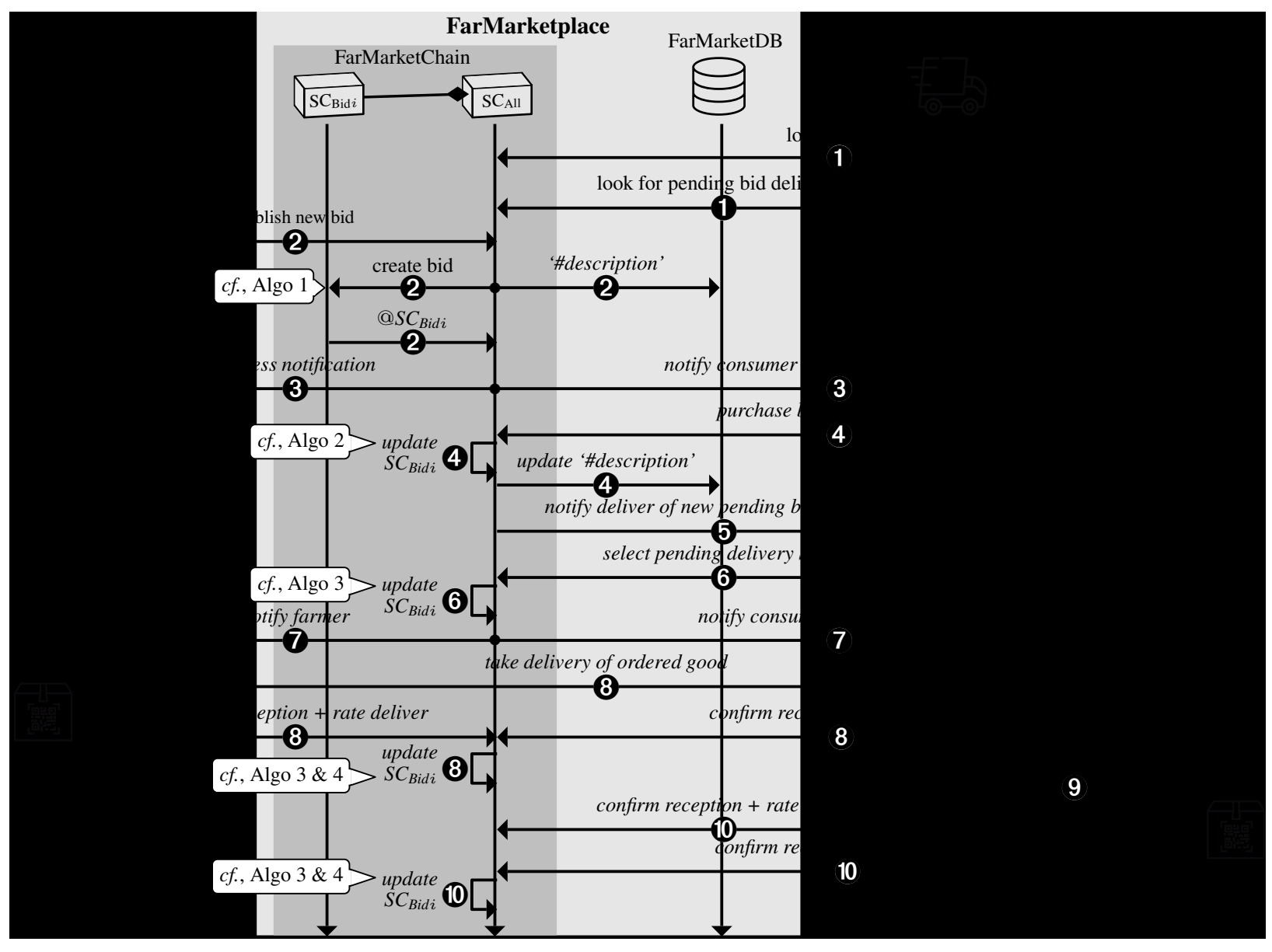

Figure 4: Messaging protocol supporting FarMarket ecosystem

consumer has sufficient money in her/his digital wallet. 454 The amount of money required to purchase the contract 455 should be equal to the bid price plus the service deliv- 456 ery price, which is denoted by $P_{\text {Del }}$. Note that $P_{\text {Del }}$ is an input of the purchase function, meaning that it is com- 457 puted outside the smart contract ${ }^{9}$ and then added to the ${ }^{458}$ corresponding state variable ( $c f$., line 3 of Algorithm 2). 459 If these conditions are satisfied, the following state vari- 460 ables are updated: (i) contract's subscriber/beneficiary ${ }^{461}$ with the consumer's ID; (ii) contract delivery price; (iii) 462 consumer's location, which is part of the bid descrip- 463 tion; (iv) contract balance credited with the required 464 amount; and (v) contract state set to WaitForDeliverer. ${ }^{465}$ In addition, the balance of the consumer's wallet is up- 466 dated accordingly ( $c f$. , line 7 in Algorithm 2). Once the ${ }^{467}$

${ }^{9}$ The function for computing the service delivery price is beyond the scope of this paper; however, it can be computed on the basis of parameters such as the consumer's location and type of goods to be 471 delivered contract state has been updated to WaitForDeliverer, deliverers who have subscribed to pending bid delivery offers are notified $(c f$., (5).

When a deliverer selects a pending bid delivery offer (cf., 6 ), the delivery function of $\mathrm{SC}_{\mathrm{Bid} i}$ is executed, as detailed in Algorithm 3. This function/algorithm verifies the contract state. If state is WaitForDeliverer, then the deliverer becomes the official delivery service provider (cf., line 2 in Algorithm 3), the contract state becomes WaitForDelivery, and both the consumer and farmer are notified of the deliverer's identity $(c f ., \boldsymbol{\nabla})$. At this stage, the deliverer must take delivery of the ordered goods $(c f ., 8)$. At the moment of exchanging the goods, the deliverer and farmer must both confirm the successful reception, which in practice, results in the call of the delivery_SC $\mathrm{Bid}_{\mathrm{Bid}}$ function, leading to a change in the contract state to OnDelivering ( $c f$., lines 4-5 in Algorithm 3). In the final stage, the deliverer delivers the goods to the consumer ( $c f$., $\boldsymbol{9})$. Both confirm the 
Table 2: State and Input variables related to FarMarket Application

\begin{tabular}{|c|c|c|}
\hline & Variable & Description \\
\hline \multirow{4}{*}{ 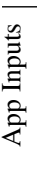 } & $\mathrm{ID}_{\text {User }}$ & Jser identifier referring either to a farmer, deliverer, or consumer, respectively denoted by $\mathrm{ID}_{\mathrm{Far}}, \mathrm{ID}_{\mathrm{Del}}$, orID $\mathrm{Ion}$ \\
\hline & $\mathrm{D}_{U \text { salance }}^{\text {bala }}$ & Balance of user's wallet \\
\hline & Amount & Amount paid by a consumer to purchase a given contract/bid denoted by $\mathrm{SC}_{\mathrm{Bid} i}$ \\
\hline & $\mathrm{P}_{\mathrm{Far}}, \mathrm{P}_{\mathrm{Del}}$ & $\begin{array}{l}\text { Prices of (i) agricultural goods to be paid to the farmer computed using the priceBid() function, which takes as inputs: product } \\
\text { type and quantity and (ii) service delivery that depends, among other inputs, on the distance between consumer and farmer }\end{array}$ \\
\hline \multirow{8}{*}{ 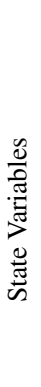 } & Desc & $\begin{array}{l}\text { Description stored in the database (see DB in Figures } 3 \text { and 4), consisting of: (i) farmer's and consumer's location denoted } \\
\text { by } \operatorname{loc}^{\mathrm{ID}} \text { Far and loc }{ }^{\mathrm{ID}} \text { Con } \text {, respectively; (ii) type; (iii) quantity of the agricultural goods; and (iv) additional comments }\end{array}$ \\
\hline & $R_{U \text { Uer } 1 \rightarrow \text { User } 2}$ & $\begin{array}{l}\text { Rating score referring to how satisfied User } 1 \text { is regarding the 'service' delivered by (or the behavior of) User } 2 \text {. All possible } \\
\text { rating score combinations are contained in a set denoted by } \mathcal{R}=\left\{R_{\mathrm{Far} \rightarrow \mathrm{Del}}, R_{\mathrm{Del} \rightarrow \mathrm{Far}}, R_{\mathrm{Del} \rightarrow \mathrm{Con}}, R_{\mathrm{Con} \rightarrow \mathrm{Far}}, R_{\mathrm{Con} \rightarrow \mathrm{Del}}\right\}\end{array}$ \\
\hline & $\mathrm{SC}_{\text {Bidi }}^{\text {state }}$ & $\begin{array}{l}\text { State variable referring to the state of contract } \mathrm{SC}_{\mathrm{Bid} i} \text { at a given point in time. Possible states are }\{\text { Available, WaitForDeliverer, } \\
\text { WaitForDelivery, OnDelivery, Delivered }\}\end{array}$ \\
\hline & $\mathrm{SC}_{\mathrm{Pidi}}^{\mathrm{ID}}$ & State variable referring to a given stakeholder $\left(c f ., \mathrm{ID}_{\text {User }}\right)$ \\
\hline & 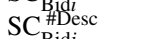 & State variable referring to the hash of the bid's description ( $c f$. , "Desc"), obtained using the priceBid(...) function \\
\hline & $\mathrm{SC}_{\text {Bidi }}^{\text {balance }}$ & State variable referring to the balance of the smart contract/bid $i$ \\
\hline & $\mathrm{SC}_{\mathrm{Bid} i}^{\mathrm{P}_{\mathrm{Far}}}, \mathrm{SC}_{\mathrm{Bid} i}^{\mathrm{P}_{\mathrm{Del}}}$ & State variables referring to prices to be paid to the farmer and deliverer $\left(c f ., \mathrm{P}_{\mathrm{Far}}, \mathrm{P}_{\mathrm{Del}}\right)$ \\
\hline & $\mathrm{SC}_{\mathrm{Bidi}}^{R} \mathrm{Rser}_{\mathrm{U}}^{\mathrm{u} \text { User2 }}$ & State variable referring to the satisfaction rating scores previously described $(c f ., \mathcal{R})$ \\
\hline
\end{tabular}

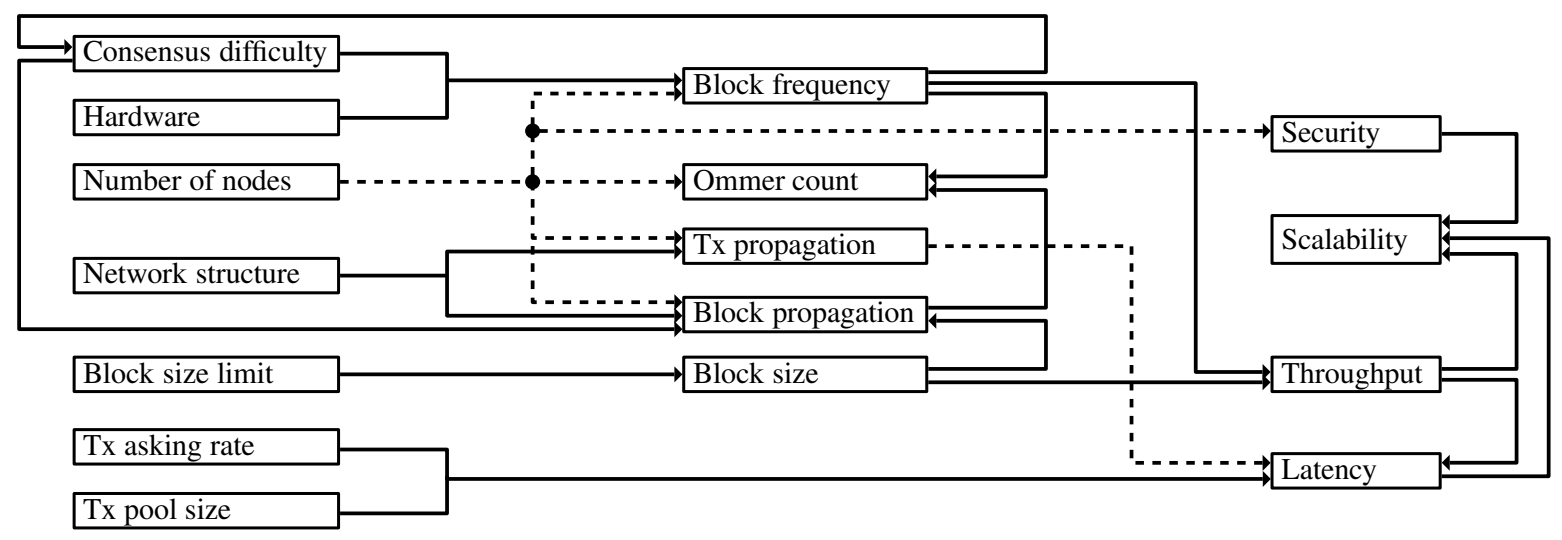

Figure 5: Performance interaction models regarding blockchain- and infrastructure-related parameters (note, that there is no difference between the solid and dashed arrows, they are used for figure clarity only)

successful delivery/reception ( $c f$. ., (1)), which leads to a ${ }_{489}$ change in the contract state to Delivered (cf., lines 6-7 in Algorithm 3), following which payments are made to ${ }^{490}$ the farmer and deliverer, and the balances updated ac- ${ }^{491}$ cordingly (cf., lines 8-10).

To provide stakeholders with the possibility of evalu- ${ }_{495}$ ating service quality, as previously discussed in Section 496 3.1 , another function is defined in the smart contract to 497 make satisfaction scores immutable. This function is 498 detailed in Algorithm 4, allowing consumers, farmers, 499 and deliverers to evaluate each other through a rating 500 score denoted by $R_{U \operatorname{ser} 1 \rightarrow \text { User } 2}(c f$. Table 2$)$. These rating 501 scores refer to the reputation/satisfaction level related 502 to a given FarMarketplace's stakeholder. Note that the 503 functions used for computing these rating scores are not 504 included in the scope of this paper.

\subsection{Ecosystem-related Key Performance Indicators}

As discussed in Section 2, only limited interactions between blockchain- and infrastructure-related parameters are formalized in the literature, although it is essential to have a comprehensive understanding of such interactions to address the QoS requirements. It is clearly not that simple to develop a unique model/representation of such interactions, as there could be as many models as there are blockchain technologies (e.g., because of different consensus mechanisms). In this section, we attempt to clarify, in a graphical manner in Figure 5, the parameter interactions of PoW-based blockchain technologies. The parameters listed on the left side of the figure correspond to application-specific parameters (e.g., implemented network architecture, number of nodes/users), whereas the parameters in the center of the figure refer to features that are specific/intrinsic 

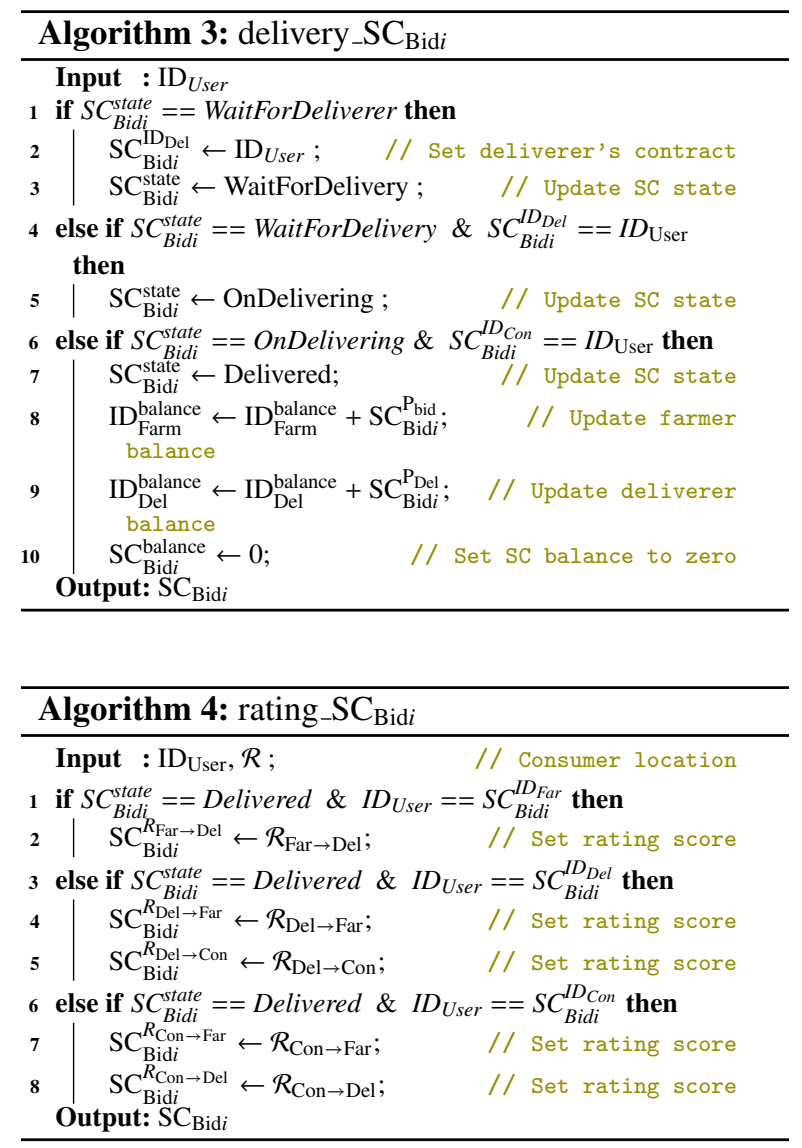

to the implemented blockchain technology (i.e., non- ${ }^{557}$ configurable parameters); the parameters on the right 558 side refer to QoS performance metrics. An arrow from 559 a frame $A$ to a frame $B$ indicates that parameter $A$ has an 560 influence, to a greater or lesser degree, on parameter $B \quad 561$ (or performance metric $B$ ). The following discusses the 562 identified interactions.

First, the "consensus difficulty" lies in the complex- ${ }^{564}$ ity of generating a block in the chain. It is known in the 565 literature that the time required to solve this challenge 566 is linked to the computational power of the network, ${ }^{567}$ which is composed of the "number of computational 568 nodes" and associated "hardware" resources (e.g., al- 569 located threads, memory, processors) (Pierro, 2019). These three parameters (consensus difficulty, hardware, number of nodes) inevitably influence the "Block (generation) frequency" parameter, as emphasized in Figure 5 .

The "Network structure", which includes data distri- 571 bution mechanisms, regroups parameters that influence 572 the delay for broadcasting transactions and blocks in the 573 chain. High block propagation delays, associated with 574 high block generation frequency, increase the likelihood of forming concurrent blocks in the blockchain network nodes. Such concurrent blocks, which are called "ommer" (or "uncle") blocks in Ethereum, could possibly not be included in the main chain.

The "number of nodes" in the blockchain network has an influence on the overall system performance as it influences the block and transaction propagation process. Indeed, the greater the number of nodes, the greater the number of messages to be propagated over the network. Another important interaction to be aware of is between the "number of nodes" and "security", as the greater the number of nodes, the greater the level of security. This interaction applies not only to PoW-based blockchain technologies, but also to technologies using BFT-like consensus.

The "Block size" parameter, which is limited by the "Block size limit" set at the configuration stage (e.g., gas limit in Ethereum), has a direct influence on the "block propagation" parameter, as well as on the system "throughput" performance. As highlighted in Figure 5, throughput is tightly coupled with the block generation frequency and block size parameters, as the product of both results in the memory throughput where transactions are written.

The latency in blockchain networks is directly dependent on the throughput parameter, although it can be negatively influenced by high transaction propagation delays. Furthermore, as discussed in Section 2.2, if the throughput is less than the transaction asking rates, congestion effects occur, resulting in an increase in latency.

Given the above discussion, we claim in this paper that it is of the utmost importance to evaluate what level of performance a given blockchain-based system, such as the proposed FarMarket ecosystem, can achieve/support in the long run. In this study, we are particularly interested in identifying the maximum achievable throughput when the blockchain is in a steady state and not saturated (which would inevitably contribute to an increase in latency in such cases). This throughput limit is referred to as the (long-run) capacity in this study and is experimentally studied in the next section.

\section{Implementation and Performance Evaluation}

The FarMarket ecosystem and associated building blocks (i.e., FarMarketchain, FarMarketplace, FarMarketApp) were implemented for experimental and evaluation purposes. The Ethereum platform was used for 


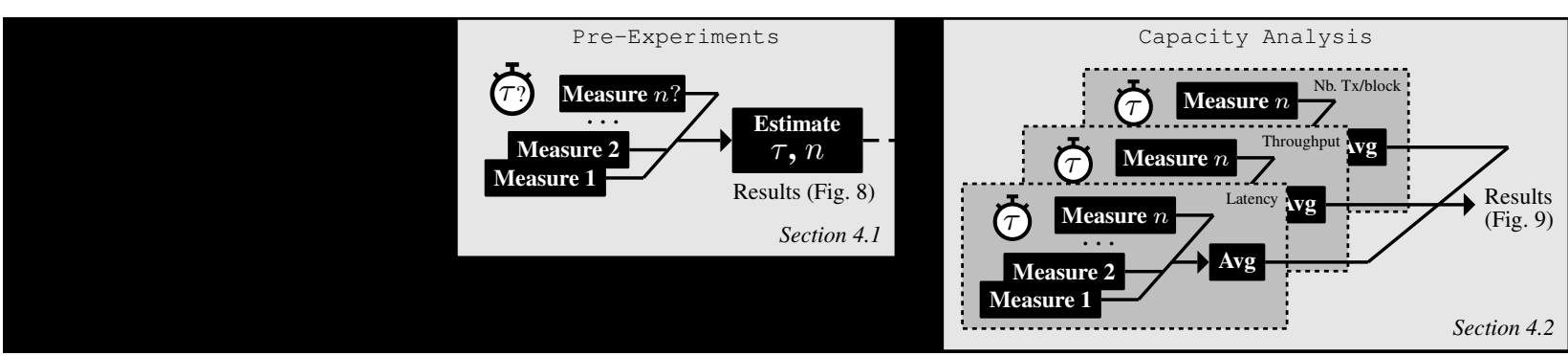

Figure 6: Methodology for fixing design of experiments

integrating the set of smart contracts ${ }^{10}$

An overview of the experimental methodology is displayed in Figure 6. A pre-experiment stage was performed to analyze and estimate the experimental settings including the appropriate number and duration of experiments to be performed. A second stage was then performed to experimentally evaluate the QoS offered by the overall ecosystem, with a focus on latency and throughput performance metrics, in addition to the number of transactions per block. These two stages are presented in Sections 4.1 and 4.2, respectively. The experimental results are further analyzed and discussed in Section 4.3, underlining the relation between the maximal throughput offered by the chain (i.e., the long-run capacity limit) and block size.

\subsection{Selection and configuration of the benchmark}

In Ethereum, different feedback controllers are im- 610 plemented to balance the security/robustness (related to 611 the computational cost) of the blockchain and QoS - ${ }^{612}$ mainly in terms of throughput and latency - offered to 613 support smart contracts. In fact, the hashing power di- 614 rectly influences the time to resolve a block, i.e., the 615 delay of mining a block, which by definition, influences 616 the latency. In this respect, in Ethereum, the difficulty 617 in mining blocks (a statistical estimate of the number of 618 hashes that must be generated to find a valid solution) is 619 re-targeted over time to control this mining delay. $\quad 620$

Figure 7 highlights the long-term evolution (over a $12{ }^{621}$ hour period) of the difficulty in our setup. It can be ob- 622 served that the difficulty is frequently re-targeted; how- 623 ever, it tends to converge towards an asymptote. In fact, 624 the system responds by increasing/decreasing the diffi- 625 culty if the previous blocks are generated faster/slower 626 than a specified mining block time, which ranges from ${ }^{627}$ 9 to 17 seconds (Pierro, 2019).

\footnotetext{
${ }^{10}$ Solidity codes of the contracts are publicly available at the following URL: https://github.com/inpprenable/FarMarketplace, last access 63 Apr. 2020
}

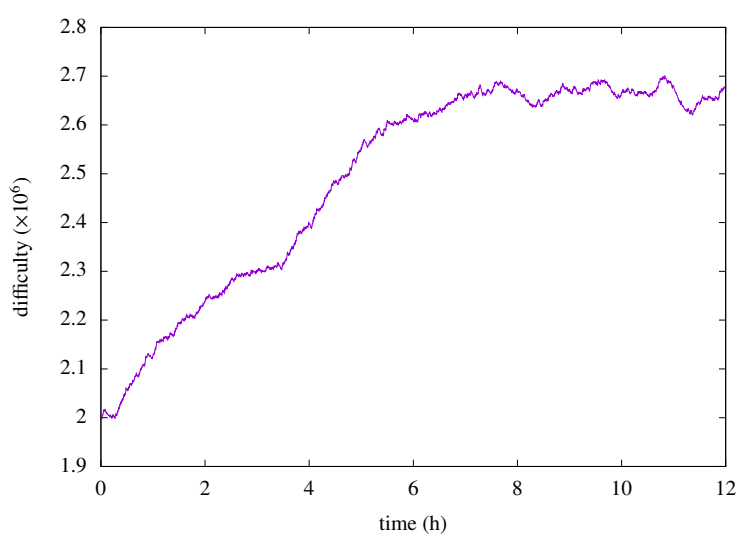

Figure 7: (Mining) difficulty per timestamp blocks

Compared with other state-of-the-art research works, our performance evaluation experiments focus on longrun QoS performance, i.e., when QoS no longer varies because of feedback control. To accelerate the control and avoid response time issues, the initial difficulty of the blockchain genesis block is set directly to its longrun value at the steady state. This value corresponds to $10 \times \#_{\text {total }}$, where $\#_{\text {total }}$ corresponds to the sum of each hashrate (the number of hashes realized by a node every second) of the computers in the network. Compared with other studies, this also allows us to focus on the real capacity of the chain and to mitigate the difference in hardware resources (e.g., number of threads, memory allowed, processors). This focus corresponds to the implementation of the proposed smart contract in Solidity (v0.5.1) on a chain shared with three computers running with Geth 1.9.6 on Ubuntu 18. The three nodes are defined as miners, one being responsible for generating the transactions. Because the latency and throughput are both influenced by block propagation delays ( $c f$., Figure 5), the nodes are connected over a switch offering a high bandwidth to maintain delays (which are noncontrollable) to a minimum, and thus allow experiments 
to be reproducible. To complete the genesis block, the 685 gas limit, which as we know influences the block size, 686 is set arbitrarily to 16777216 gas. Furthermore, to en- 687 sure that our experimental platform would demonstrate 688 the expected behavior in terms of difficulty $\left(\#_{\text {total }}\right)$ and 689 the influence of the number of threads, a preliminary 690 experimental analysis was performed, as presented in 691 Appendix A.

Based on this configuration setting, pre-experiments 693 were performed to determine the number $(n)$ and duration $(\tau)$ of the experiments to be reproduced in the second evaluation stage. As mentioned previously, the latency and throughput offered by the core chain were considered the performance metrics (both being obtained by comparing the timestamps when transactions were generated for a contract and submitted to the chain). Figure 8 displays the evolution of both metrics, in addition to the number of transactions per block. Note that the experiments were performed after a $30 \mathrm{~min}$ block generation period to ensure that stability of the difficulty was achieved.

Figure 8(a) provides insight into the evolution of the number of transactions per block for ten minutes, for ten experiments, one transaction being submitted per sec- 705 ond. It can be observed that the convergence is rela- 706 tively fast and the blockchain remains (reasonably) sta- 707 ble. This is also confirmed by observing the QoS met- ${ }^{708}$ rics, namely the latency (see Figure 8(e)) and through- 709 put (see Figure 8(c)). Throughput is computed as the 710 number of transactions for a block divided by the de- 711 lay to mine that block; latency is based on the time ${ }^{712}$ difference between the emission and validation of the 713 transaction. After ten minutes, the latency is in the ex- 714 pected range defined earlier (with a standard deviation 715 less than one second and an average mining delay of 716 11.6 seconds per block). These experiments allowed us 717 to select a simulation duration of $\tau=10 \mathrm{~min}$ for the 718 second experimental evaluation stage (a sufficient num- 719 ber of samples being available, 52 blocks on average). In a further step, the confidence was analyzed by con- 720 sidering a greater number of experiments.

Figure 8(b) displays the evolution of the average 722 number of transactions per block when experiments 723 were added. The relative error corresponds to the dif- 724 ference between the average for a given number and av- 725 erage for 30 experiments. Figures 8(d) and 8(f) provide 726 insight into the same analysis for the throughput and la- 727 tency metrics, respectively. It can be observed that the 728 steady state is achieved with ten experiments and that 729 the increase in the number of experiments does not sig- 730 nificantly change the precision. Consequently, in the 731 following, each configuration is repeated $n=10$ times. $\quad 732$
Because our main goal is to evaluate the entire ecosystem, the following section aims to define the maximal service that can be offered to support the emission of smart contracts. It is, then, important to identify the capacity offered by the chain to store contractrelated information. In this respect, the experiments are repeated not only according to the parameters identified in this section, but also by increasing the transactionemission rate.

\subsection{Analysis of the FarMarketChain Capacity}

Figure 9 displays the evolution of the three metrics previously identified; however, this time for a given transaction-emission rate ranging from $1 \mathrm{Tx} / \mathrm{s}$ (as previous) to $21 \mathrm{Tx} / \mathrm{s}$. Each point consists of experiments of ten minutes, repeated ten times. As the gasLimit was arbitrarily chosen ( 1 blockSize $=16777216$ gas), we repeated the same set of experiments on another chain with a ten times greater gasLimit. Figures 9(d), 9(b), and 9(f) correspond to this second experiment.

\subsubsection{Number of transactions per block}

Figures 9(a) and 9(b) provide insight into the evolution of the number of transactions inside a block according to the transactions emission rate. Two behaviors emerge before and after the emission throughput value that we refer to as capacity. Before the capacity is achieved, the evolution of the number of transactions per block is linear and corresponds to the average delay of mining a block (it varies, yet is experimentally near 11 seconds) multiplied by the emission rate. Once the capacity is surpassed, the delay required to fill a block is less than the delay of mining a block. This results in the filling of the blocks with the maximum number of transactions, leading to an approximately constant number of transactions per block, with minimal variation between the two experiments.

\subsubsection{Latency}

Figures 9(e) and 9(f) display the average latency of the transactions according to the throughput. We can again extract two behaviors: before and after the capacity. With an emission rate less than the capacity, as the block is not filled, the transaction is validated and inserted into the next block when it reaches a node. The latency is thus equal to the time required to wait for the next block, which corresponds to the mining delay. This delay differs minimally between the two experiments (owing to the feedback control of the difficulty); hence, the latency differs only marginally. After the capacity, the system is overloaded. As the validation throughput 


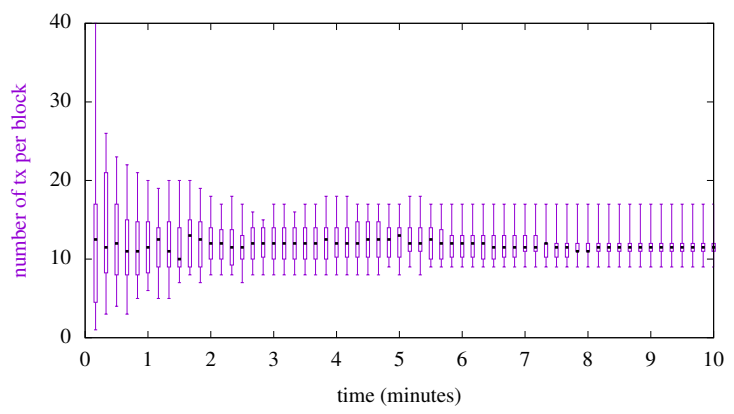

(a) Number of Tx per Block $(/ \tau=10 \mathrm{~min})$

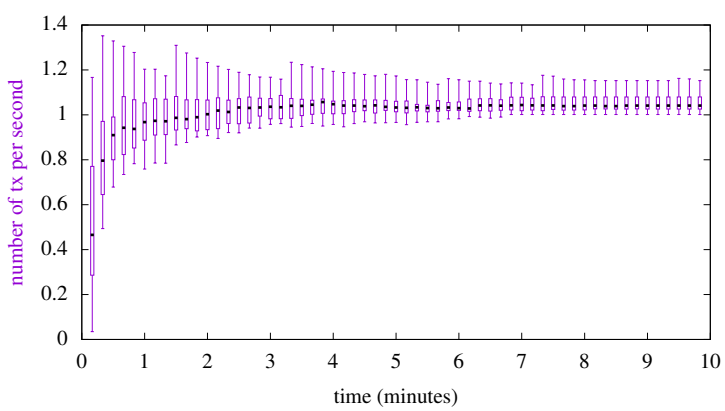

(c) Throughput $(/ \tau)$

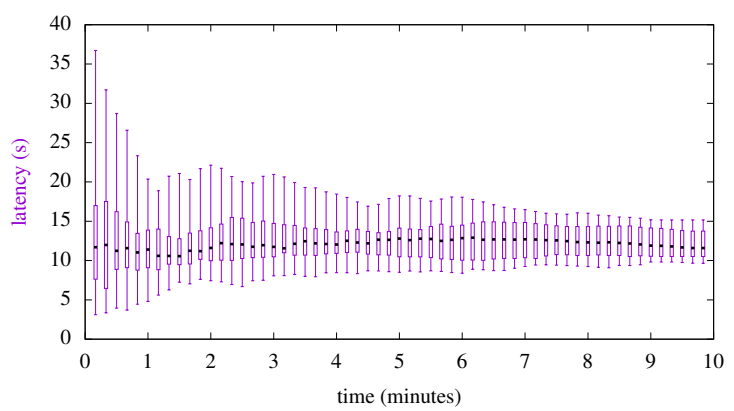

(e) Latency $(/ \tau)$

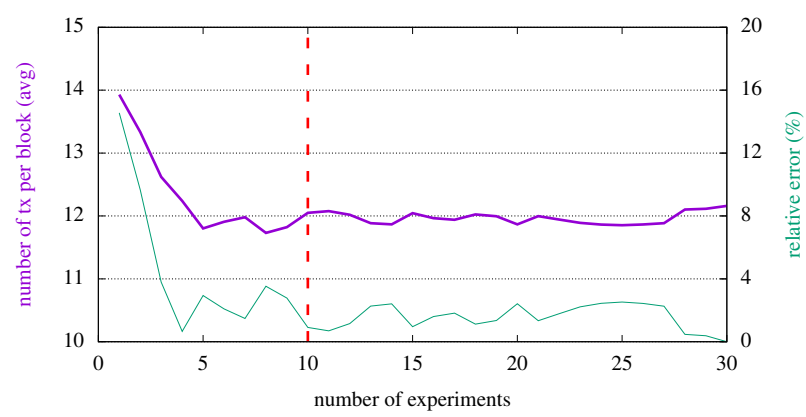

(b) Number of Tx per Block $(/ n)$

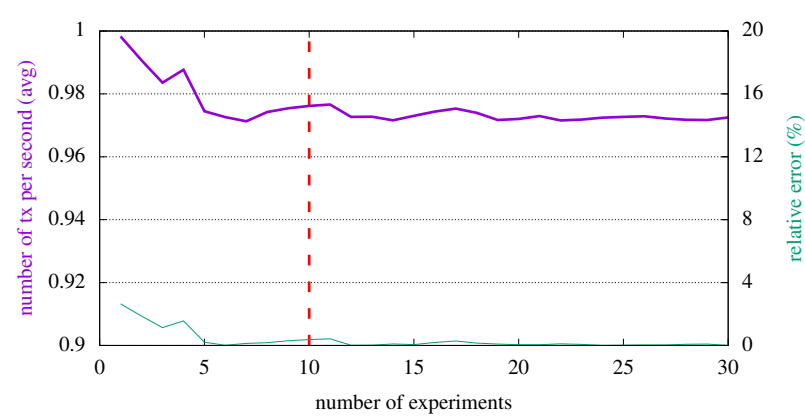

(d) Throughput $(/ n)$

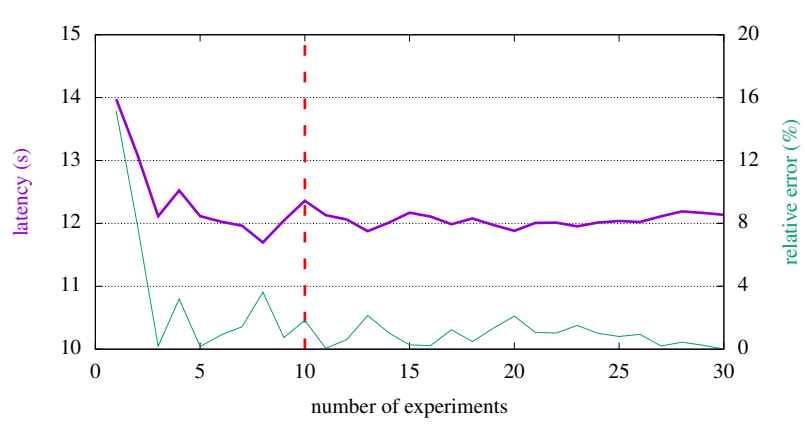

(f) Latency $(/ n)$

Figure 8: Evolution of QoS metrics for different number of experiments $(n)$ and in time $(\tau)$

is less than the emission rate, the transaction is queued, 747 leading to a significant increase in the latency.

\subsubsection{Throughput}

Figures 9(c) and 9(d) provide insight into the evolu- 751 tion of the validation throughput. In the first part, the validated throughput is equal to the emission rate be- 752 cause every emitted transaction is validated (leading to 753 the identity function, with minimal differences between 754 the two experiments). In the second part, blocks are sat- 755 urated such that the validated throughput is limited to a 756 constant, which corresponds to the capacity and is de- 757 fined by the ratio between the number of transactions 758 per block and the delay of mining a block.

As assumed, the number of transactions contained in 760 a block is proportional to the size of the block. By increasing the block size by a factor of 10 , we also increase the number of transactions in a block by a factor of 10. The maximal validated throughput, i.e., the $c a$ pacity, is also proportional to the block size.

\subsection{Discussions}

As evidenced through the review of the literature on blockchain-based e-agriculture solutions presented in Section 1, a large number of the research studies did not provide performance evaluation results for their solutions, and even fewer compared their solutions with other state-of-the-art approaches. In this respect, we propose to compare our proposal to another smart farming contract, the one proposed by Tian (2017). This con- 


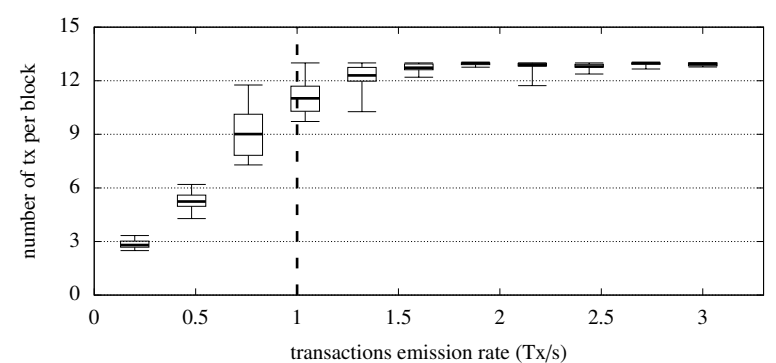

(a) Number of Tx per Block (gasLimit $\times 1$ )

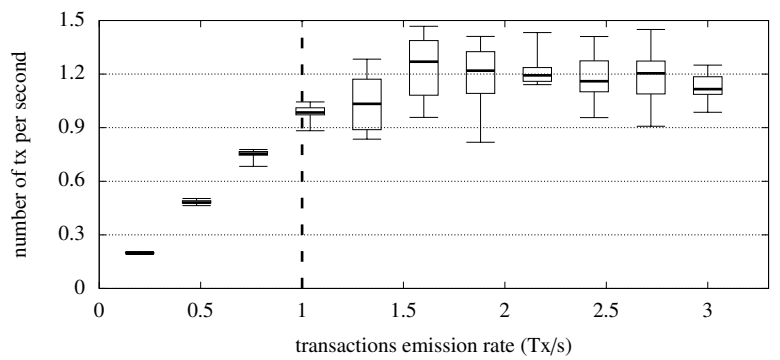

(c) Throughput (gasLimit $\times 1)$

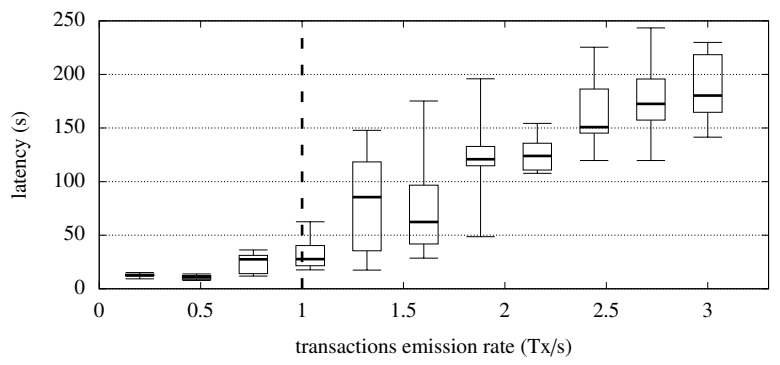

(e) Latency (gasLimit $\times 1$ )

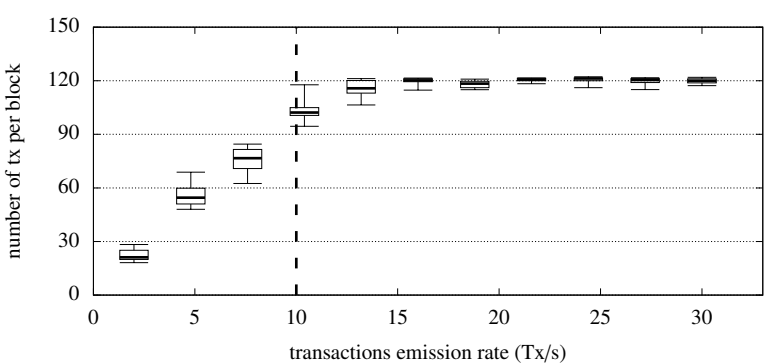

(b) Number of Tx per Block (gasLimit×10)

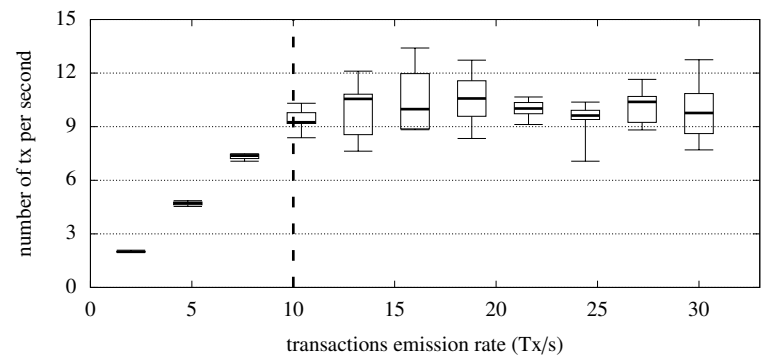

(d) Throughput (gasLimit $\times 10)$

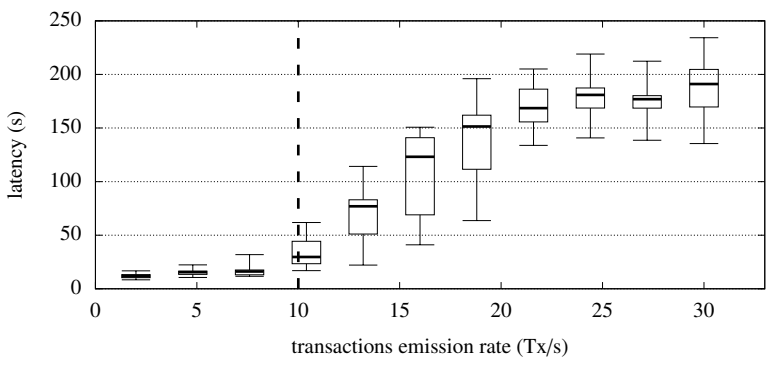

(f) Latency (gasLimit $\times 10)$

Figure 9: Experimental analysis of capacity of blockchain

tract is lighter to emit (the transaction fee is 894159 gas 777 instead of 1148305 gas as in the proposed FarMarket- 778 related contracts), which should result in filling blocks 779 with a greater number of contracts.

This is experimentally confirmed/validated in Figure 10 considering measurements of the capacity for 782 different block sizes (defined in terms of gasLimit) 783 and linear regressions between these values, stating the 784 linearity assumed in the previous section. It can be ob- 785 served that the slope rate is lower (by 19\%) for the pro- 786 posed approach compared with Tian (2017)'s smart con- 787 tract, which is in line with the fact that the proposed 788 contract is $28 \%$ heavier in gas transaction cost. This 789 supports a link between the transaction cost of a contract 790 and the slope (i.e., the number of transactions per unit of 791 block size). Given this, a deeper analysis could provide 792 a prediction of the block size required to achieve a given throughput, for a given contract, such that the emission transaction rate remains below the blockchain capacity. In the implementation of Ethereum, the block size can be tuned by modifying the gasLimit of the blocks.

However, it is important to remember that increasing the block size can increase the propagation delays, and therefore, specific attention must be considered to ensure that the network capacity is sufficient. Furthermore, because the capacity linearly depends on the block generation throughput, it is also related to the delay in mining a block. By reconfiguring the feedback control of the difficulty (i.e., by modifying the blockchain implementation), such a delay can be adapted to support the expected capacity. Here, specific attention must be considered as it could promote the ap- 


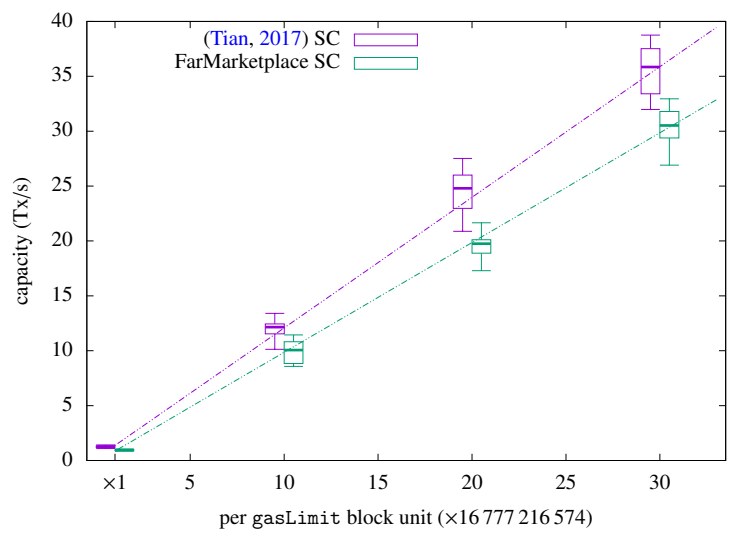

Figure 10: Comparison of capacity evolution for two smart contracts

pearance of ommers through the network. Finally, this ${ }^{841}$ capacity can be increased by optimizing the contract (i.e., by making it lighter). From a macroscopic perspective, the complexity of the algorithm writing data to the chain should be as low as possible (Wood, 2017). Using an optimizer such as GASOL (Albert et al., 2020) is an acceptable option to reduce contract gas fees in this respect.

\subsection{Approach limitations}

Even though the experiments were performed in a ${ }_{852}$ state near the steady regime for the mining delay, the ${ }_{853}$ network considered in this study was not subject to 854 high network latency or data corruption. When using 855 blockchain over the Internet, this delay could be more 856 significant (e.g., approximately 12.6 seconds consider- ${ }_{857}$ ing the Bitcoin chain (Decker and Wattenhofer, 2013)), 858 which in certain applications could lead to end-to-end ${ }_{859}$ latency and throughput problems as discussed in (Fan et al., 2020; Bez et al., 2019). One of the major im- 860 pacts of larger delays is the higher probability of the 861 appearance of ommers related to the desynchronization 862 effect. The feedback control on delay would respond 863 by increasing the difficulty, and thus the delay of min- 864 ing a block, which explains why the mining delay of the 865 main Ethereum blockchain is approximately $14.4 \mathrm{sec}-866$ onds (Pierro, 2019).

We also stress the fact that in this study we did not ${ }_{868}$ consider the potential evolution of the gasLimit. In- 869 deed, miners could be interested in increasing this limit 870 ( to decrease the number of blocks to mine) such that the 871 capacity would evolve as defined previously. Clients 872 such as Geth implement the limitation of the variation 873 between two blocks as defined in (Wood, 2017) (ap- 874 proximately $0,1 \%$ ). For 10 minute experiments (i.e., 875 with an average of 52 blocks), it could correspond up to a $5 \%$ increase in the block size.

\section{Conclusion, implications, and limitations}

\subsection{Conclusion}

Research initiatives on how to integrate agriculture with blockchain technology remain in their infancy, with several outstanding research challenges and gaps (Hang et al., 2020; Zhao et al., 2019). Among these, as revealed in the literature review of the research presented in this paper, there is a requirement for blockchain-based farming marketplaces that support the trading of agricultural goods between farmers and interested third party stakeholders (e.g., food transformation companies, retailers), which should motivate a movement away from the centralized and monopolistic model that shapes today's food value chain.

This study introduced such a blockchain-based farming marketplace, called "FarMarketplace", a part of a larger ecosystem referred to as "FarMarket" In this respect, trading (smart) contract templates between farmers, interested third-party consumers, and deliverers were specified. In addition to the specification of the FarMarket ecosystem, a comprehensive methodology was introduced to assist software solution integrators to better understand (and measure) what QoS performance a FarMarket-like ecosystem could achieve and support in the long run. A particular focus was given to the maximum achievable throughput (Tx/s) in the long run, which is referred to as capacity in this study. The experimental analyses presented in this paper should lead to interesting discussions regarding the critical aspects/interactions to be considered between blockchain- and infrastructure-related parameters.

\subsection{Implications}

This research presented three main theoretical implications. First, it contributes to the literature on smart farming (or e-agriculture) by proposing a thorough state-of-the-art approach for the use of blockchain technology, identifying the trends and gaps in the current research.

Secondly, it contributes to making agricultural and farming practices more sustainable in two respects: (i) it facilitates the emergence of local agriculture markets, thus encouraging agriculture and food sourcing and (ii) the nature of blockchain technology helps to prove that climate friendly requirements are met, as farmers are facing an increasing number of obligations for monitoring, verifying, and reporting according to sustainability requirements. 
Thirdly, it contributes to the software development ${ }_{923}$ community. To the best of our knowledge, there is 924 only limited research work that thoroughly discusses 925 the interactions between blockchain- and infrastructure- 926 related parameters, and how they influence the overall ${ }_{927}$ (end-to-end) QoS performance. The experimental eval- 928 uation of the maximum achievable throughput (Tx/s) in 929 the long run (i.e., capacity) is a contribution of this re- 930 search work.

\subsection{Limitations and Future research directions}

Several limitations of our work should be addressed and discussed. The first limitations, related to our experiments, were identified and discussed in Section 4.4; therefore, we refer the reader to that section for experiment-related limitations).

A second limitation relates to the proposed smart contract templates, and particularly to the fact that the set of data items considered in our templates could possibly not cover all the trading requirements for the different types of agricultural goods/markets to be sold/purchased. Even though the Description parameter introduced as part of our smart contracts is sufficiently generic to be extended with any new information that the farmer/seller could deem as relevant (only the hash of the Description is added to the smart contract), it would be convenient to adopt standardized metadata for describing agricultural goods for enhanced interoperability. Semantic- or ontology-based approaches could be investigated and combined with blockchain-based farming ecosystems (Bacco et al., 2019; Lokers et al., 2016).

Although beyond the scope of this research, one key challenge lies in the adoption of blockchain-based solutions by small and medium businesses. The reason for this is twofold: (i) they frequently lack the expertise to invest in blockchain (a common argument that can be found in the literature is that there is no significant adoption of blockchain technology outside of cryptocurrencies) and (ii) clear feedback on the experience gained from the deployment of blockchain-based systems is limited owing to its recent emergence, although selected reports have provided predictions on the potential gains; see, e.g., IBM report ${ }^{11}$ that states that blockchain can reduce the time required to trace the source of food from seven days to 2.2 seconds. It is therefore imperative to make blockchain infrastructures affordable and easy to use in the near future.

\footnotetext{
${ }^{11}$ https://newsroom.ibm.com/How-Blockchain-Could-Mend-OurFractured-Global-Food-Supply-Chain
}

Data privacy and security aspects related to blockchain have not been discussed significantly in this paper, although they are of importance in blockchain applications. Indeed, by design, data inserted into a blockchain cannot be erased. Furthermore, the strength of a public blockchain is that everyone can download and verify blocks and transactions, thus leaving room for privacy concerns. Although sensitive data in the proposed solution are stored apart from the blockchain (in an external database called FarMarketDB), the "hash" of that data is added to the blockchain (via smart contract). Other more advanced solutions could be explored in the future, similar to the ones proposed in (Kosba et al, 2016; Bünz et al, 2019).

Our study of the parameters' influence is also limited by the chosen blockchain; Ethereum runs on the Geth client. In fact, the diversity of consensus protocols and chain parameters supporting existing blockchain technologies makes it difficult to objectively compare two technologies. To do this effectively, our analysis should be extended to other chains that function with other consensus protocols. With this extension, a similar comparison basis could be defined, enabling a better choice for the blockchain infrastructure selection.

Finally, we must highlight the fact that the relation considered in Section 4.1 between "difficulty" and "Blocktime" is based on an assumption (this assumption being further detailed in Appendix A), and further research should be performed to determine more accurate numerical values (e.g., regarding the average difficulty calculation).

\section{Acknowledgments}

This work was partly supported by the French PIA project "Lorraine Université d'Excellence" reference ANR-15-IDEX-04-LUE. We would like to express special thanks to Mario Lezoche for his contribution to the brainstorming challenges and ideas related to blockchain-based farming.

\section{Appendix A. Preliminary benchmark analysis}

In this paper, certain relations were assumed, as in Section 4.1. This appendix proposes an explanation of these relations based on a probabilistic model of Ethereum nodes. This model has been subjected to experiments to verify the consistency of our benchmark platform. Finally, a relation that establishes the time and difficulty required to mine a block is discussed. 


\section{Appendix A.1. Difficulty and Blocktime Relation}

In the context of Ethereum, the difficulty-related feedback control relies on an assumed relation given in (A.1), where $\tau$ is the average blocktime (i.e., the average delay to mine a block), $d$ is the difficulty of the chain (relying on a statistical estimate of the number of hashes that must be generated to find a valid solution to mine a block), and $\#_{t o t}$ is the network hashrate (i.e., the number of hashes realized by a node on a per second basis). As the blocktime is fixed to approximately ten seconds in Ethereum, this relation is frequently simplified by $d=10 \times \#_{\text {tot }}$ (cf., Section 4.1).

$$
\tau=\frac{d}{\#_{t o t}}
$$

This relation can be interpreted as the mean of an exponential distribution of parameter $\lambda=\frac{\#_{t o t}}{d}$. Indeed, as the mining of the block is comparable to a brute force ${ }^{1005}$ attack for a puzzle solution in a set sufficiently large, the 1006 search of the solution can be modeled by a continuous 1007 memoryless distribution. This model also provides an 1008 explanation of the hashrate additivity property, as a net- 1009 work composed of $N$ nodes results in a system of $\left(n_{i}\right)_{i \in N}{ }^{1010}$ independent nodes seeking the solution to the puzzle, ${ }^{1011}$ each having a given hashrate denoted by $\#_{i}$. Therefore, 1012 for each node $n_{i}$, the random value denoted by $X_{i}$ for ${ }^{1013}$ finding a solution follows an exponential parameter distribution $\frac{\#_{i}}{d}$. The network's random value $X_{\text {tot }}$ to find a solution among all network nodes is therefore equal to $X_{\text {tot }}=\min \left(\left\{X_{i}\right\}_{i \in N}\right)$ as a solution is found if and only if one node solves the puzzle, following an exponential network distribution $\frac{\sum_{i \in N} \#_{i}}{d}$. Overall, the hashrate of a network can be determined by summing the hashrate of the entire network.

\section{Appendix A.2. Validity of the model}

To verify the consistency of the proposed model and benchmark platform, the relation between blocktime and hashrate was tested. To achieve this, a blockchain with the same initial parameters (including the same difficulty) was performed with a variable number of hashrates. The experiment duration (ten minutes) was sufficiently short to neglect the change in difficulty due to the feedback control. In the experiment that produced the maximum number of blocks, which is more likely to be influenced by this control, 64 blocks were produced, which could modify the difficulty in Ethereum accord- ${ }^{1014}$ ing to A.3 by up to $3 \%$ (this equation is further detailed in the next section). Indeed, according to A.3, a block can modify the difficulty of a block by a factor $\frac{1}{2048}$. Thus, after 64 blocks, $\Delta d=\left|\left(1 \pm \frac{1}{2048}\right)^{64}-1\right|<3.2 \%$.

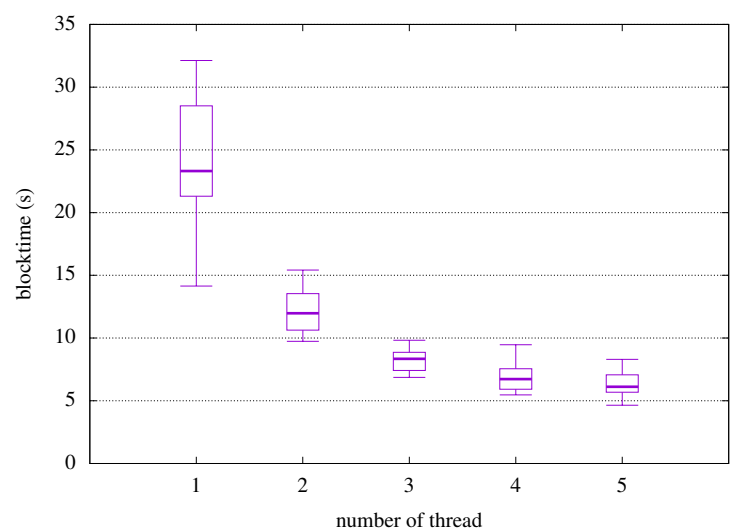

Figure A.11: Evolution of average blocktime according to number of threads

Furthermore, to eliminate the influence of the transfer time factor, the blockchain was executed on a single machine and the hashrate variation was performed by changing the number of threads allocated to the mining. Every thread used one core of the machine and proceeded independently via multi-threading. As long as the threads did not compete among themselves, they could be considered as having the same hashrate as they were executed on a similar core.

Given this hypothesis, the problem is equivalent to the relation given in A.2, where $\#_{\text {thread }}$ refers to the hashrate of a single thread that is assumed to be constant, and $n b_{\text {thread }}$ to the number of threads used. Figure A.11 provides insight into the results obtained for an experiment realized ten times per number of threads. The relation between the blocktime and number of threads was inversely proportional. Figure A.12 stresses this finding by indicating a linear relation between the inverse of the delay and number of threads, following a linear regression with $\rho=0.91$. When considering a large number of threads, this relation becomes obsolete owing to the fact that threads began to compete with each other on the same machine.

$$
\tau=\frac{d}{\#_{\text {thread }} \times n b_{\text {thread }}}
$$

\section{Appendix A.3. Difficulty Definition}

The definition of the difficulty of a block used in the current version of Ethereum has been extracted from yellow paper (Wood, 2017), and is given in (A.3) $\forall n>$ 


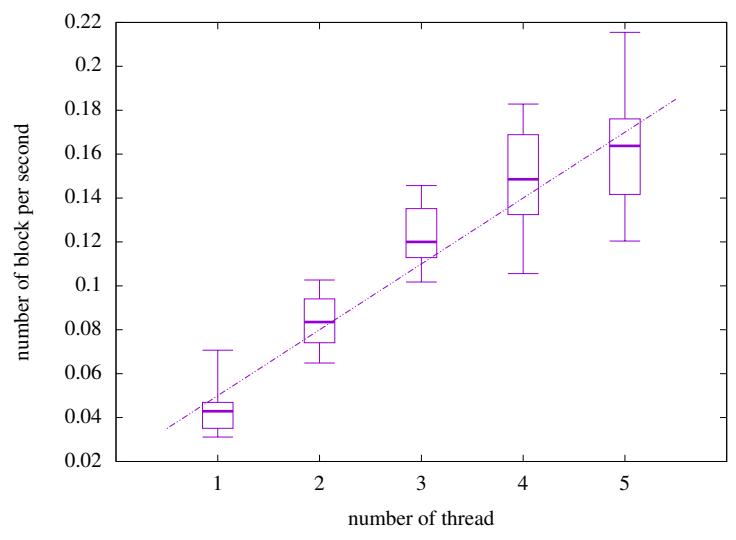

Figure A.12: Evolution of inverse of average blocktime according to ${ }^{1058}$ number of threads

0 :

$$
\begin{aligned}
& H_{d}^{n}=\max \left(H_{d}^{0}, H_{d}^{n-1}+\right. \\
&\left.\left\lfloor\frac{H_{d}^{n-1}}{2048}\right\rfloor \times \max \left(y-\left\lfloor\frac{H_{t}^{n}-H_{t}^{n-1}}{9}\right\rfloor,-99\right)+\epsilon\right) \begin{array}{r}
1066 \\
1067 \\
1068
\end{array} \\
& y=\left\{\begin{array}{lll}
1069 \\
1 & \text { if } H^{n-1} \text { has no ommers } & \\
2 & \text { otherwise }, & 1070 \\
1064
\end{array}\right. \\
&
\end{aligned}
$$

where $H^{n}$ refers to a block with a sequence number de- ${ }^{1073}$ noted by $n, H_{d}^{n}$ is the difficulty of that block, $H_{t}^{n}$ is the ${ }^{1074}$ timestamp (in seconds) when the block was generated, ${ }_{1076}^{1075}$

epsilon is the "difficulty bomb" designed to force users 1078 to update their chain (note that a high number of blocks ${ }^{1079}$ - approximately 5000000 - must be considered to ac- ${ }^{1080}$ tivate such a "bomb"), and $y$ is a term depending on the ${ }_{1082}$ appearance of ommers in the previous block. The max- ${ }^{1083}$ imum functions included in the formula ensure that the ${ }^{1084}$ difficulty does not fall below the original value, while ${ }_{1086}^{1085}$ limiting its evolution speed. This relation can be inter- 1087 preted as explained by (Wood, 2017): before the diffi- ${ }^{1088}$ culty bomb, if no ommer appears, and if the delay be- ${ }^{1089}$ tween two blocks is between 9 and 18 seconds, the dif- ${ }_{1091}{ }^{1090}$ ficulty does not change. However, if the blocktime is 1092 not in this interval, the difficulty must be reduced or in- ${ }^{1093}$ creased to favor the next blocktime in that interval.

\section{References}

Blandford, D., 2019. Supply Response to Increased Demand for Food. ${ }^{1099}$ Global Challenges For Future Food And Agricultural Policies, 1, 1100 61.

Zhao, G., Liu, S., Lopez, C., Lu, H., Elgueta, S., Chen, H., Boshkoska, ${ }^{1102}$ B. M., 2019. Blockchain technology in agri-food value chain man- 1103 agement: A synthesis of applications, challenges and future re- ${ }^{1104}$ search directions. Computers in Industry, 109, 83-99.
Rabah, K., 2018. Convergence of AI, IoT, big data and blockchain: a review. The Lake Institute Journal, 1(1), 1-18.

Lin, Y. P., Petway, J. R., Anthony, J., Mukhtar, H., Liao, S. W., Chou, C. F., Ho, Y. F., 2017. Blockchain: The evolutionary next step for ICT e-agriculture. Environments, 4(3), 50.

Aker, J. C., Ghosh, I., Burrell, J., 2016. The promise (and pitfalls) of ICT for agriculture initiatives. Agricultural Economics, 47(S1), 35-48.

Lezoche, M., Hernandez, J., Diaz, M. D. M. A., Panetto, H., Kacprzyk, J. (2020). Agri-food 4.0: a survey of the supply chains and technologies for the future agriculture. Computers in Industry, 116, 103187.

Wolfert, S., Ge, L., Verdouw, C., Bogaardt, M. J., 2017. Big data in Smart Farming - a review. Agricultural Systems, 153, 69-80.

Vermesan, O., Friess, P., 2016. Digitising the industry-internet of things connecting the physical, digital and virtual worlds. River Publishers.

Krishnan, R. S., Julie, E. G., Robinson, Y. H., Raja, S., Kumar, R., Thong, P. H., 2020. Fuzzy Logic based Smart Irrigation System using Internet of Things. Journal of Cleaner Production, 252, 119902.

Kamble, S. S., Gunasekaran, A., Gawankar, S. A., 2020. Achieving sustainable performance in a data-driven agriculture supply chain: A review for research and applications. International Journal of Production Economics, 219, 179-194.

Klerkx, L., Jakku, E., Labarthe, P., 2019. A review of social science on digital agriculture, smart farming and agriculture 4.0: New contributions and a future research agenda. NJAS-Wageningen Journal of Life Sciences, 100315.

Rose, D. C., Chilvers, J., 2018. Agriculture 4.0: Broadening responsible innovation in an era of smart farming. Frontiers in Sustainable Food Systems, 2, 87.

Pinto, M. M. A., Kovaleski, J. L., Yoshino, R. T., Pagani, R. N., 2019. Knowledge and technology transfer influencing the process of innovation in green supply chain management: A multicriteria model based on the DEMATEL Method. Sustainability, 11(12), 3485.

Tripoli, M., Schmidhuber, J., 2018. Emerging Opportunities for the Application of Blockchain in the Agri-food Industry. FAO and ICTSD: Rome and Geneva. Licence: CC BY-NC-SA, 3.

Bermeo-Almeida, O., Cardenas-Rodriguez, M., Samaniego-Cobo, T., Ferruzola-Gḿez, E., Cabezas-Cabezas, R., Bazán-Vera, W., 2018. Blockchain in agriculture: A systematic literature review. In: International Conference on Technologies and Innovation, Guayaquil, Ecuador, November, 44-56.

Feng, H., Wang, X., Duan, Y., Zhang, J., Zhang, X., 2020. Applying blockchain technology to improve agri-food traceability: A review of development methods, benefits and challenges. Journal of Cleaner Production, 121031.

Kamilaris, A., Fonts, A., Prenafeta-Boldú, F. X., 2019. The rise of blockchain technology in agriculture and food supply chains. Trends in Food Science \& Technology, 91, 640-652.

Zheng, Z., Xie, S., Dai, H. N., Chen, X., Wang, H., 2018. Blockchain challenges and opportunities: A survey. International Journal of Web and Grid Services, 14(4), 352-375.

Panarello, A., Tapas, N., Merlino, G., Longo, F., Puliafito, A., 2018. Blockchain and IoT integration: A systematic survey. Sensors, 18(8), 2575.

Nowiński, W., Kozma, M., 2017. How can blockchain technology disrupt the existing business models?. Entrepreneurial Business and Economics Review, 5(3), 173-188.

Al-Jaroodi, J., Mohamed, N., 2019. Blockchain in industries: A survey. IEEE Access, 7, 36500-36515.

Juma, H., Shaalan, K., Kamel, I., 2019. A Survey on Using Blockchain in Trade Supply Chain Solutions. IEEE Access, 7, 184115-184132. 
Xu, X., Weber, I., Staples, M., 2019. Case Study: AgriDigital. In: 1171 Architecture for Blockchain Applications. Springer, Cham, 239- 1172 255

Dujak, D., Sajter, D., 2019. Blockchain applications in supply chain. 1174 In: SMART supply network. Springer, Cham, 21-46.

Hang, L., Ullah, I., Kim, D. H., 2020. A secure fish farm platform 1176 based on blockchain for agriculture data integrity. Computers and 1177 Electronics in Agriculture, 170, 105251.

Galvez, J. F., Mejuto, J. C., Simal-Gandara, J., 2018. Future chal- 1179 lenges on the use of blockchain for food traceability analysis. TrAC 1180 Trends in Analytical Chemistry, 107, 222-232.

Thomason, J., Ahmad, M., Bronder, P., Hoyt, E., Pocock, S., 1182 Bouteloupe, J., Donaghy, K., Huysman, D., Willenberg, T., 1183 Joakim, B., 2018. Blockchain - Powering and empowering the 1184 poor in developing countries. In: Transforming climate finance and 1185 green investment with blockchains. Academic Press, 137-152. 1186

Bumblauskas, D., Mann, A., Dugan, B., Rittmer, J. (2020). A 1187 blockchain use case in food distribution: Do you know where your 1188 food has been?. International Journal of Information Management, 1189 52,102008

1190

Pinna, A., Ibba, S., 2018. A blockchain-based Decentralized System 1191 for proper handling of temporary Employment contracts. In: Sci- 1192 ence and information conference. Springer, Cham, 1231-1243. 1193

Devi, M. S., Suguna, R., Joshi, A. S., Bagate, R. A., 2019. Design of 1194 IoT Blockchain Based Smart Agriculture for Enlightening Safety 1195 and Security. In: International Conference on Emerging Technolo- 1196 gies in Computer Engineering. Springer, Jaipur, India, February, 1197 7-19.

Patil, A. S., Tama, B. A., Park, Y., Rhee, K. H., 2017. A framework for 1199 blockchain based secure smart green house farming. In: Advances 1200 in Computer Science and Ubiquitous Computing. Springer, 1162- 1201 1167.

Tse, D., Zhang, B., Yang, Y., Cheng, C., Mu, H., 2017. Blockchain 1203 application in food supply information security. In: IEEE Interna- 1204 tional Conference on Industrial Engineering and Engineering Man- 1205 agement. Singapore, Singapore, December, 1357-1361.

Davcev, D., Kocarev, L., Carbone, A., Stankovski, V., Mitresk, K., 1207 2018. Blockchain-based Distributed Cloud/Fog Platform for IoT 1208 Supply Chain Management. In 8th international conference on ad- 1209 vances in computing, electronics and electrical technology. Kuala 1210 Lumpur, Malaysia, February, 51-58.

Lucena, P., Binotto, A. P., Momo, F. D. S., Kim, H., 2018. A case 1212 study for grain quality assurance tracking based on a Blockchain 1213 business network. arXiv preprint arXiv:1803.07877.

Mao, D., Hao, Z., Wang, F., Li, H., 2019. Novel automatic food trad- 1215 ing system using consortium blockchain. Arabian Journal for Sci- 1216 ence and Engineering, 44(4), 3439-3455.

Tian, F., 2017. A supply chain traceability system for food safety 1218 based on HACCP, Blockchain \& Internet of Things. In: Inter- 1219 national conference on service systems and service management. 1220 Dalian, China, June, 1-6.

Bore, N., Kinai, A., Waweru, P., Wambugu, I., Mutahi, J., Kemunto, 1222 E., Weldemariam, K., 2020. ADW: Blockchain-enabled Small- 1223 scale Farm Digitization. arXiv preprint arXiv:2003.06862. 1224

Stefanova, M., Salampasis, M., 2019. Farm-to-fork traceability: 1225 Blockchain meets agri-food supply chain. In: 12th European Fed- 1226 eration for Information Technology in Agriculture, Food and the 1227 Environment. Rhodes, Greece, June.

Leng, K., Bi, Y., Jing, L., Fu, H. C., Van Nieuwenhuyse, I., 2018, Research on agricultural supply chain system with double chain 1230 architecture based on blockchain technology. Future Generation 1231 Computer Systems, 86, 641-649.

Kumar, M. V., Iyengar, N. C. S., 2017. A framework for Blockchain 1233 technology in rice supply chain management. Advanced science 1234 and technology letters, 146, 125-130.
Iqbal, R., Butt, T. A., 2020. Safe farming as a service of blockchainbased supply chain management for improved transparency. Cluster Computing, 1-12.

George, R. V., Harsh, H. O., Ray, P., Babu, A. K., 2019. Food quality traceability prototype for restaurants using blockchain and food quality data index. Journal of Cleaner Production, 240, 118021

Caro, M. P., Ali, M. S., Vecchio, M., Giaffreda, R., 2018. Blockchainbased traceability in Agri-Food supply chain management: A practical implementation. In 2018 IoT Vertical and Topical Summit on Agriculture-Tuscany. Borgo San Luigi, Italy, May, 1-4.

Surasak, T., Wattanavichean, N., Preuksakarn, C., Huang, S. C. H., 2019. Thai Agriculture Products Traceability System using Blockchain and Internet of Things. Editorial Preface From the Desk of Managing Editor, 10(9), 15.

Malik, S., Kanhere, S. S., Jurdak, R., 2018. Productchain: Scalable blockchain framework to support provenance in supply chains. In: 17th IEEE International Symposium on Network Computing and Applications. Cambridge, USA, October, 1-10.

Hua, J., Wang, X., Kang, M., Wang, H., Wang, F. Y., 2018. Blockchain based provenance for agricultural products: A distributed platform with duplicated and shared bookkeeping. In: IEEE Intelligent Vehicles Symposium IV. Changshu, Suzhou, China, June, 97-101.

Shakhbulatov, D., Arora, A., Dong, Z., Rojas-Cessa, R., 2019. Blockchain Implementation for Analysis of Carbon Footprint across Food Supply Chain. In: IEEE International Conference on Blockchain. Atlanta, USA, July, 546-551.

Lin, J., Shen, Z., Zhang, A., Chai, Y., 2018. Blockchain and IoT based food traceability for smart agriculture. In: 3rd International Conference on Crowd Science and Engineering. Vancouver, Canada, July, 1-6.

Reddy, G. B., Kumar, K. R., 2020. Quality Improvement in Organic Food Supply Chain Using Blockchain Technology. In: Innovative Product Design and Intelligent Manufacturing Systems. Springer, Singapore, 887-896.

Xie, C., Sun, Y., Luo, H., 2017. Secured data storage scheme based on blockchain for agricultural products tracking. In: 3rd International Conference on Big Data Computing and Communications. Yonago, Japan, July, 45-50.

Papa, S. F., 2017. Use of Blockchain technology in agribusiness: transparency and monitoring in agricultural trade. In: International Conference on Management Science and Management Innovation. Suzhou, China, June.

Awan, S. H., Ahmed, S., Safwan, N., Najam, Z., Hashim, M. Z., Safdar, T. Role of Internet of Things (IoT) with Blockchain Technology for the Development of Smart Farming. Journal of Mechanics of Continua and Mathematical Sciences, 14, 170-188.

Laux, C. M., Hurburgh Jr, C. R., 2012. Using quality management systems for food traceability. Journal of Industrial Technology, 26(3), 1.

Udokwu, C., Kormiltsyn, A., Thangalimodzi, K., Norta, A., 2018. The state of the art for blockchain-enabled smart-contract applications in the organization. In: Ivannikov Ispras Open Conference. Moscow, Russia, November, 137-144.

Xie, J., Tang, H., Huang, T., Yu, F. R., Xie, R., Liu, J., Liu, Y., 2019. A survey of blockchain technology applied to smart cities: Research issues and challenges. IEEE Communications Surveys \& Tutorials, 21(3), 2794-2830.

Sajana, P., Sindhu, M., Sethumadhavan, M., 2018. On blockchain applications: Hyperledger fabric and Ethereum. International Journal of Pure and Applied Mathematics, 118(18), 2965-2970.

Ali, M. S., Vecchio, M., Pincheira, M., Dolui, K., Antonelli, F., Rehmani, M. H., 2018. Applications of blockchains in the Internet of Things: A comprehensive survey. IEEE Communications Surveys \& Tutorials, 21(2), 1676-1717. 
Xiao, Y., Zhang, N., Lou, W., Hou, Y. T., 2020. A survey of distributed consensus protocols for blockchain networks. IEEE Communications Surveys \& Tutorials. DOI: 10.1109/COMST.2020.2969706

239 Vukolić, M., 2015. The quest for scalable blockchain fabric: Proofof-work vs. BFT replication. In: International workshop on open problems in network security. Zurich, Switzerland, October, 112125.

Pierro, G. A., Rocha, H., 2019. The influence factors on ethereum transaction fees. In: 2nd IEEE/ACM International Workshop on Emerging Trends in Software Engineering for Blockchain. Montreal, Canada, May, 24-31.

Wood, G., 2014. Ethereum: A secure decentralised generalised transaction ledger. Ethereum project yellow paper, 151(2014), 1-32.

Albert, E., Correas, J., Gordillo, P., Román-Díez, G., Rubio, A., 2020. GASOL: Gas analysis and optimization for Ethereum smart contracts. In: International Conference on Tools and Algorithms for the Construction and Analysis of Systems. Dublin, Ireland, 118125.

Decker, C., Wattenhofer, R., 2013. Information propagation in the bitcoin network. In: IEEE International Conference on Peer-to-Peer Computing. Trento, Italy, September, 1-10.

Bacco, M., Barsocchi, P., Ferro, E., Gotta, A., Ruggeri, M., 2019. The Digitisation of Agriculture: a Survey of Research Activities on Smart Farming. Array, 3, 100009.

Lokers, R., Knapen, R., Janssen, S., van Randen, Y., Jansen, J., 2016. Analysis of Big Data technologies for use in agro-environmental science. Environmental Modelling \& Software, 84, 494-504.

Fan, C., Ghaemi, S., Khazaei, H. \& Musilek, P, 2020. Performance Evaluation of Blockchain Systems: A Systematic Survey. Access, $8,126927-126950$

Bez, M., Fornari, G. \& Vardanega, T. The scalability challenge of ethereum: An initial quantitative analysis.

Kosba, A., Miller, A., Shi, E., Wen, Z. \& Papamanthou, C. Hawk: The Blockchain Model of Cryptography and Privacy-Preserving Smart Contracts.

Bünz, B., Agrawal, S., Zamani, M. \& Boneh, D. Zether: Towards Privacy in a Smart Contract World.

Sumathi, M. \& Sangeetha, S.: Blockchain Based Sensitive Attribute Storage and Access Monitoring in Banking System.

Singh, Nikita \& Vardhan, Manu: Distributed Ledger Technology based Property Transaction System with Support for IoT Devices.

Ramli, Carroline Dewi Puspa Kencana \& Nielson, Hanne Riis \& Nielson, Flemming: The logic of XACML. Science of Computer Programming, 83 .

Di Francesco Maesa, Damiano \& Mori, Paolo \& Ricci, Laura: Blockchain Based Access Control.

Esposito, Christian \& Ficco, Massimo \& Gupta, B B: Blockchainbased authentication and authorization for smart city applications. 